\title{
Antiderivative Antialiasing Techniques in Nonlinear Wave Digital Structures
}

\author{
DAVIDE ALBERTINI, AES Student Member, ALBERTO BERNARDINI, AND AUGUSTO SARTI \\ (davide.albertini@polimi.it) \\ (alberto.bernardini@polimi.it) \\ (augusto.sarti@polimi.it)
}

Dipartimento di Elettronica, Informazione e Bioingegneria (DEIB), Politecnico di Milano, 20133 Milano, Italy

\begin{abstract}
The Wave Digital Filter (WDF) formalism is becoming a popular approach for the digital emulation of audio circuits. Nonlinear WDFs, like other kinds of discrete-time nonlinear filters used in Virtual Analog modeling applications, are often affected by aliasing distortion. Recently formalized Antiderivative Antialiasing (ADAA) methods are capable of significant aliasing reduction even with low oversampling factors. This paper discusses different strategies to integrate $p$ th-order ADAA methods into stateful WDFs with a single one-port or multiport nonlinearity while preserving the modularity property typical of traditional WDFs. The effectiveness of the proposed approach is verified by applying the discussed ADAA techniques to three nonlinear audio circuits containing diode-based nonlinearities and a BJT transistor.
\end{abstract}

\section{INTRODUCTION}

In the past two decades, considerable research effort has been made to develop accurate and computationally lightweight Virtual Analog (VA) implementations of nonlinear audio circuits [1-8]. Many techniques have been proposed in the literature for the digital emulation of analog synthesizers [9-11], distortion pedals [12], and audio amplifiers $[13,14]$ that contain nonlinear circuit elements such as diodes [15-20], transistors [21], transformers [22], and tubes [23]. However nonlinear discrete-time systems, such as those encountered in VA modeling, may be subjected to aliasing distortion. This issue may occur whenever a bandlimited input signal is processed by a nonlinear function, which adds to the signal spectrum additional frequency components that overcome the Nyquist frequency and are mirrored into the signal base-band as distortion artifacts, causing inharmonicity, beating, and heterodyning [24].

High oversampling factors are commonly used as a trivial solution to reduce aliasing. However increasing the sampling frequency may lead to computationally inefficient implementations that could violate real-time execution constraints. Hence alternative less expensive antialiasing methods are desirable, and many techniques have been proposed in the VA literature, dealing with discrete-time oscillators for subtractive synthesis [24, 25], emulations of clipping stages used in overdrive or distortion circuits [26, 27], and interpolation filters combined with decimation filters for nonlinear waveshaping [28].

Recently a novel antialiasing approach applicable to a large class of nonlinear functions, called Antiderivative Antialiasing (ADAA), has been introduced by Parker et al. in [29]. The method approximates the input signal as a continuous-time piecewise linear function, applies the nonlinear function to it, and then convolves the resulting signal with the continuous-time impulse response of a low-pass filter before sampling it back to the digital domain. Ultimately these steps yield a less-aliased nonlinear function approximation expressed as a combination of its antiderivatives. In [29], however, just first and second-order approximations based on antiderivatives are discussed. Subsequently Bilbao et al. in [30] extended the method employing higher-order antiderivatives and reframed the approach as the repeated differentiation of a $p$ th-order antiderivative of the nonlinear function.

Nonetheless ADAA presents two major drawbacks: a low-pass filtering effect and the introduction of a fractional delay of $p / 2$ samples [31]. The first limitation can be easily overcome through mild oversampling or by designing a simple linear filter. The additional introduced delay, however, becomes problematic in systems having feedback paths. For this reason ADAA has been applied almost exclusively to memoryless systems [29, 30, 32]. An extension to stateful systems has been proposed by Holters in 
[33] and consists of a global parameter modification to the coefficient matrices of the state-space formulation to compensate for the additional delay introduced in the system by the ADAA filter.

Inspired by the approach in [33], in a previous work [34] we introduced a different methodology for applying $p$ th-order ADAA to stateful Wave Digital Filters (WDFs) with a single one-port nonlinearity. Such a methodology allows us to exploit the inherent modularity property of WDFs in the design of ADAA algorithms for VA modeling. This article is an extended version of [34], incorporating an exhaustive overview of ADAA techniques applicable to Wave Digital (WD) one-port nonlinearities. Moreover we discuss a method for the application of ADAA to a WD multi-port nonlinear element (the BJT).

The paper is structured as follows. Sec. 1 briefly revises the structure of WDFs with one nonlinearity. Sec. 2 proposes a novel method to integrate first-order ADAA [29] into stateful WDFs with a single one-port or multi-port nonlinear element. Sec. 3 generalizes the method to higherorder ADAA. Sec. 4 discusses different generic models of WD one-port nonlinearities and the corresponding ADAA approximations. WD ADAA models of specific one-port and multi-port nonlinearities, i.e., the single exponential diode, a pair of diodes in antiparallel and the BJT, are then presented in Secs. 5 and 6. The effectiveness of the proposed ADAA approach in the WD domain is verified in three examples of application discussed in Sec. 7. Sec. 8 concludes this paper and proposes possible future developments.

\section{BRIEF OVERVIEW ON WDF MODELING}

WDF theory was first introduced by A. Fettweis in the 1970s and later reorganized in [35] as a methodology to model reference analog circuits as digital filters, based on networks of input-output blocks characterized by scattering relations and communicating through port connections. In particular a WDF is a port-wise lumped model of a reference circuit that detaches the topological information from the models of circuit elements. The reference topology is described through scattering junctions, called adaptors, enforcing Kirchhoff continuity laws, while circuit elements are derived from the lumped discretization of their constitutive equations. A peculiarity of WDFs is the use of the so-called wave variables obtained from Kirchhoff variables (i.e., current and voltage) through a linear and invertible transformation. At one port of a circuit element, voltage wave variables are defined as

$$
a=v+Z i \quad b=v-Z i,
$$

with inverse mapping

$$
v=\frac{a+b}{2} \quad i=\frac{a-b}{2 Z},
$$

where $v$ is the port voltage, $i$ is the port current, $a$ is the wave incident to the element, $b$ is the wave reflected from the element, and $Z$ is an additional free parameter, called reference port resistance. This free parameter is set to adapt linear circuit elements, thus obtaining explicit WD scatter-
Table 1. Wave mappings of common linear one-ports.

\begin{tabular}{lccc}
\hline \hline Element & Constit. eq. & Wave mapping & Adapt. cond. \\
\hline Res. Sour. & $v=E_{g}+R_{g} i$ & $b[k]=E_{g}[k]$ & $Z=R_{g}$ \\
Resistor & $v=R i$ & $b[k]=0$ & $Z=R$ \\
Capacitor & $i(t)=C \frac{\mathrm{d} v(t)}{\mathrm{d} t}$ & $b[k]=a[k-1]$ & $Z=\frac{T_{s}}{2 C}$ \\
Inductor & $v(t)=L \frac{\mathrm{d} i(t)}{\mathrm{d} t}$ & $b[k]=-a[k-1]$ & $Z=\frac{2 L}{T_{s}}$ \\
\hline
\end{tabular}

ing relations in the discrete-time domain in which the reflected wave does not depend on the incident wave. In this way local delay-free-loops arising from the port connections of elements to WD junctions are eliminated. Moreover continuous-time derivatives in constitutive equations of dynamic elements (e.g., capacitors and inductors) are typically approximated using the trapezoidal discretization method. Adapted WD models of capacitors and inductors become one-sample delay elements (with a sign flip for inductors). The constitutive equations in the continuous-time Kirchhoff domain, discrete-time scattering relations in the WD domain, and corresponding adaptation conditions of most common linear one-port elements are reported in Table 1 , where $T_{\mathrm{s}}=1 / f_{\mathrm{s}}$ is the sampling period defined as the reciprocal of the sampling frequency $f_{\mathrm{s}}$.

On the other hand connection networks embedding the topological information of the reference circuits are implemented in the WD domain using scattering junctions (adaptors) characterized by scattering matrices [35]. General formulas for computing the scattering matrices that model arbitrary reciprocal or nonreciprocal connection networks are discussed in [36-41].

\subsection{WDFs With Connection Tree Structure}

In order to ensure the computability of a WD structure the corresponding signal-flow diagram should not contain any delay-free loop [35, 40, 42, 43]. Sarti et al. [44] proposed a systematic method to implement circuits containing up to one nonlinear element using explicit WD structures, which avoid the use of iterative solvers. The approach in [44] is applicable to WD networks with a Binary Connection Tree (BCT) structure. A BCT is characterized by one (nonlinear) element as root, an interconnection of series/parallel three-port junctions as nodes, and adapted linear one-port elements as leaves. Each three-port junction is adapted toward the root, which means that all junction ports facing other junctions (i.e., other nodes) or the nonlinear element (i.e., the root) are made reflection free by properly setting the corresponding free parameters.

In light of the recent advances in the WD modeling of reciprocal [39] and non-reciprocal [38] junctions, the BCT concept can easily be generalized to the concept of Connection Tree (CT), whose root is a nonlinear element, nodes can be adaptors with $N \geqslant 2$ ports, and leaves are adapted linear elements. The root can also be a $J$-port nonlinearity to which $J>1$ different subtrees are connected [3, 17, $21,23,45-48]$. Under the assumption that no topological delay-free-loops are present, a CT can also be implemented in an explicit fashion. 


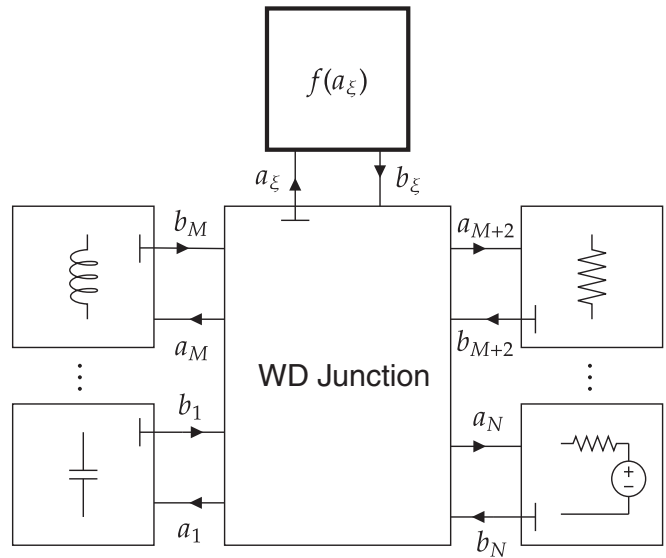

Fig. 1. Single-junction WDF with one nonlinear element.

The evaluation of a CT consists of three main phases. At first the forward scan phase is performed. It consists of traversing the CT from the leaves to the root. At each sampling step the waves reflected from adapted linear one-port elements in each subtree are known, according to the wave mappings in Table 1. Therefore it is possible to compute all the waves reflected from the junctions at their adapted ports, performing the scattering operations in the correct order (i.e., the computational flow goes from the leaves to the root). The second phase consists of the local root scattering stage, in which the waves reflected by the (nonlinear) element at the root are evaluated. Lastly the backward scan stage consists of traversing the tree structure from the root to the leaves until all the waves incident to the linear adapted elements are computed.

In the following sections we will show how ADAA techniques can be applied to WDFs with a CT structure.

\section{FIRST-ORDER ADAA IN NONLINEAR WDFS}

\subsection{One-Port Nonlinearity Case}

Let us consider the generic WDF in Fig. 1 characterized by a single (reciprocal or nonreciprocal) $N$-port WD junction. $N-1$ linear one-ports and one nonlinear element are connected to the junction. Since the WDF in Fig. 1 has a CT structure (i.e., the nonlinear element is the root, the linear elements are the leaves, and the node is the $N$-port junction), it can be implemented in an explicit fashion using the procedure, based on forward scan, local root scattering, and backward scan, discussed in the previous section. Depending on the reference circuit, the single node can be decomposed into an interconnection of nodes without delay-free-loops (e.g., BCTs or CTs with multiple nodes). However it is worth noticing that we do not lose generality by describing the topological information with a single node. The scattering matrix $\mathbf{S}$ of the WD $N$-port junction can be computed, given the $N$ free parameters $Z_{1}, \ldots, Z_{N}$, according to the formulas presented in $[38,39]$. The port of the WD junction facing the nonlinear element is made reflection free by choosing the free parameter at that port in such a way that the corresponding diagonal entry of the scattering matrix $\mathbf{S}$ goes to zero.
Matrix $\mathbf{S}$ relates incident and reflected waves at a sampling time step $k$ as

$$
\mathbf{a}[k]=\mathbf{S b}[k],
$$

Waves incident to the elements are collected into the column vector $\mathbf{a}[k]=\left[a_{1}[k], \ldots, a_{N}[k]\right]^{T}$. We assume that the WD one-port elements are ordered as follows. Waves $a_{1}[k], \ldots$, $a_{M}[k]$ are incident to the $M<N$ linear dynamic elements (capacitors and inductors). The wave at position $M+1$ of vector $\mathbf{a}[k]$, called $a_{\xi}[k]$, is incident to the nonlinear element. Finally waves $a_{M+2}[k], \ldots, a_{N}[k]$ are incident to linear instantaneous elements (resistors and resistive sources). Therefore vector $\mathbf{a}[k]$ can be expressed as

$$
\mathbf{a}[k]=\left[a_{1}[k], \ldots, a_{M}[k], a_{\xi}[k], a_{M+2}[k], \ldots a_{N}[k]\right]^{T} .
$$

Similarly we can define the vector $\mathbf{b}[k]$ as the column vector of waves reflected by the elements and incident to the WD junction. Waves reflected by linear elements are computed according to Table 1, while the nonlinear element at the root is characterized by a scattering relation $b_{\xi}=f\left(a_{\xi}\right)$. For the moment we assume that $f\left(a_{\xi}\right)$ is an explicit function in the WD domain, available in analytic form, even though this is not always the case. In fact, as extensively discussed in Sec. 4, the element at the root may be characterized by a nonlinear implicit function.

So far we have described the WD structure prior to ADAA application. Let us now locally apply the first-order ADAA method proposed in [29] to the root nonlinear scattering relation $b_{\xi}=f\left(a_{\xi}\right)$ by substituting $f$ with the following approximation (subscript $\xi$ is omitted for better readability):

$$
\tilde{f}(a[k])=\left\{\begin{array}{lll}
\frac{F_{1}(a[k])-F_{1}(a[k-1])}{a[k]-a[k-1]} & \text { if } & a[k] \not \approx a[k-1] \\
f\left(\frac{a[k]+a[k-1]}{2}\right) & \text { if } & a[k] \approx a[k-1]
\end{array}\right.
$$

where $a[k]$ and $b[k]$ are the discrete-time wave signals and $F_{1}$ is the first-order antiderivative of $f$. Unfortunately as outlined in $[29,33]$ the first-order ADAA filter introduces halfsample delay in the digital structure, altering the temporization of the system. At each time-step $k$ the non-antialiased version of the same system would have a unitary delay that temporarily stores the state, to be used at time-step $k+1$. However the additional delay introduced by ADAA in the feedback path adds up to the unitary delays implementing capacitors and inductors, making the total delay $1.5 \mathrm{sam}-$ ples long. Therefore in addition to the altered temporization of the system ADAA introduces a misalignment in time between the signals entering the WD junction with scattering matrix $\mathbf{S}$ during the backward scan stage.

Let us consider (3) as the generic scattering operation performed at time step $k$ throughout the backward scan stage. The column vector $\mathbf{b}[k]$ of waves reflected from the elements and incident to the WD junction is given by

$$
\mathbf{b}[k]=\left[b_{1}[k], \ldots, b_{M}[k], \tilde{b}_{\xi}[k], b_{M+2}[k], \ldots, b_{N}[k]\right]^{T},
$$

where in turn $\tilde{b}_{\xi}[k]$ is defined as

$$
\tilde{b}_{\xi}[k]=\tilde{f}\left(a_{\xi}[k]\right) .
$$


Since $\tilde{f}$ introduces a half sample delay, it becomes clear that there is a misalignment in time between $\tilde{b}_{\xi}[k]$ and all the other elements of $\mathbf{b}[k]$. To synchronize the signals we apply a half-sample delay filter with Z-domain transfer function $H(z)=\frac{1}{2}\left(1+z^{-1}\right)$ to all the entries in $\mathbf{b}[k]$ but $\tilde{b}_{\xi}[k]$. It is worthwhile noticing that such a filter $H(z)$ acts as the ADAA filter (4) in the linear case; hence to some extent we are applying the same antialiasing filter even to the linear elements of the system. Thus we obtain a synchronized version of (3), expressed as

$$
\begin{aligned}
\tilde{\mathbf{a}}[k] & =\mathbf{S} \tilde{\mathbf{b}}[k] \\
\tilde{\mathbf{b}}[k] & =\left[\tilde{b}_{1}[k], \ldots, \tilde{b}_{M}[k], \tilde{b}_{\xi}[k], \tilde{b}_{M+2}[k], \ldots, \tilde{b}_{N}[k]\right]^{T}
\end{aligned}
$$

with

$$
\tilde{b}_{n}[k]=\frac{1}{2}\left(b_{n}[k]+b_{n}[k-1]\right),
$$

and $1 \leqslant n \leqslant N$ with $n \neq \xi$. The half-sample delay filter applied to each entry of $\mathbf{b}[k]$, with the exception of $\tilde{b}_{\xi}[k]$, resolves the issue of time misalignment between the waves incident to the WD junction during the backward scan. The last open problem to be addressed is the altered timing due to the added delay. If we further expand (6), considering the waves reflected from dynamic elements, according to Table 1, we obtain

$$
\begin{aligned}
\tilde{\mathbf{b}}[k]= & {\left[ \pm \tilde{a}_{1}[k-1], \ldots,\right.} \\
& \left. \pm \tilde{a}_{M}[k-1], \tilde{b}_{\xi}[k], \tilde{b}_{M+2}[k], \ldots, \tilde{b}_{N}[k]\right]^{T}
\end{aligned}
$$

where $\tilde{a}_{n}[k-1]=\frac{1}{2}\left(a_{n}[k-1]+a_{n}[k-2]\right)$.

Equation (8) puts in evidence that the introduced halfsample delay results in a total delay of 1.5 samples in the feedback path of the system. The additional delay is compensated employing a method similar to that applied in [33] to state-space systems. Therefore the coefficients of $\mathbf{S}$ depending on the sampling period $T_{\mathrm{s}}$ are modified using an "expanded" sampling period $\tilde{T}_{\mathrm{s}}=1.5 T_{\mathrm{s}}$, since a delay of 1.5 samples corresponds to a 1 -sample delay at the reduced sampling frequency $\tilde{f}_{\mathrm{s}}=2 f_{\mathrm{s}} / 3$. Thus (3) is approximated with

$$
\mathbf{a}[k] \approx \tilde{\mathbf{S}} \tilde{\mathbf{b}}[k],
$$

where $\tilde{\mathbf{b}}[k]$ represents the vector $\mathbf{b}[k]$ after the application of the synchronization delays in (6) and (7). Matrix $\tilde{\mathbf{S}}$ is defined as $\tilde{\mathbf{S}}=\mathbf{S}\left(\tilde{T}_{\mathrm{s}}\right)$, which means that the free parameters $Z_{1}$, $\ldots, Z_{N}$ essential for the computation of the scattering matrix need to be changed according to the expanded sampling pe$\operatorname{riod} \tilde{T}_{\mathrm{s}}$. In particular, for capacitors we set $Z_{n}=\tilde{T}_{\mathrm{s}} /\left(2 C_{n}\right)$ and for inductors $Z_{n}=2 L_{n} / \tilde{T}_{\mathrm{s}}$. Finally, operating the system at its original sampling frequency $f_{\mathrm{s}}$ but with modified coefficients allows us to compensate for the additional delay, completing the integration of the ADAA method into stateful WDFs with one nonlinearity.

The discussed implementation procedure is resumed in Algorithm 1, written in pseudo-code. We assume to have a voltage source driven by the input signal $V_{\text {in }}[k]$ and connected to the $j$ th port of the WD junction. The output signal $V_{\text {out }}[k]$, instead, is the port voltage at the $l$ th port. Moreover we define $\tilde{\mathbf{S}}_{\xi}$ as the $\xi$ th row vector of matrix $\tilde{\mathbf{S}}$. In order to compute the output voltage $V_{\text {out }}[k]$ the wave incident to the lth element needs to be aligned with its reflected wave, as shown in line 10 of Algorithm 1.

It is worth noting that simple modifications to Algorithm 1 should be done in order accommodate scenarios in which current sources are present and current output signals are considered.

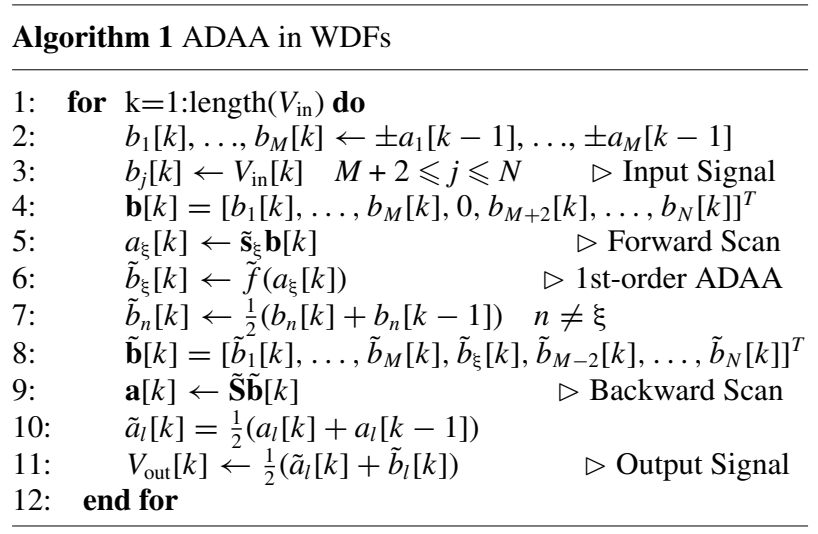

\subsection{Multi-Port Nonlinearity Case}

This section discusses the application of ADAA in WDFs characterized by a CT structure and one $J$-port nonlinear element (with $J>1$ ), e.g., a BJT, MOSFET, or vacuum tube. Let us assume for now that the WD model of the $J$ port element is characterized by an explicit vector scattering relation in the form

$$
\mathbf{b}_{\Xi}=\mathbf{f}\left(\mathbf{a}_{\Xi}\right)=\left[f_{1}\left(\mathbf{a}_{\Xi}\right), \ldots, f_{J}\left(\mathbf{a}_{\Xi}\right)\right]^{T},
$$

where $\mathbf{a}_{\Xi}=\left[a_{\xi 1}, \ldots, a_{\xi J}\right]$ is the vector of waves incident to the $J$ ports of the element, $\mathbf{b}_{\Xi}$ is the vector of waves reflected by the $J$ ports of the element, and $\mathbf{f}$ is a vector nonlinear function.

It is apparent that applying an ADAA method to (10) is not trivial, because we are dealing with multivariate nonlinear components $f_{1}\left(\mathbf{a}_{\Xi}\right), \ldots, f_{J}\left(\mathbf{a}_{\Xi}\right)$ and it is not obvious how to compute the antiderivatives. A possibility is integrating each component $f_{j}\left(\mathbf{a}_{\Xi}\right)$ with respect to a single variable, leaving the other variables fixed, similarly to that done with nonlinear multi-port nonlinearities in the Kirchhoff domain in [49]. The choice of the integration variable may seem arbitrary; however, in practical applications, certain variables could introduce more aliasing than the others to the components of the vector function $\mathbf{f}$. Whenever possible such variables should be identified and chosen as integration variables. In the following integration variables are indicated as $a_{\xi q}$, with $1 \leqslant q \leqslant J$, where $a_{\xi q}$ may vary from function to function. Therefore any component $f_{j}\left(\mathbf{a}_{\Xi}\right)$ in (10), with $1 \leqslant j \leqslant J$, given its first-order antiderivative $F_{j, 1}\left(\mathbf{a}_{\Xi}\right)$ with respect to $a_{\xi q}$, can be approximated by using 
the first-order ADAA method as

$$
\tilde{f}_{j}\left(\mathbf{a}_{\Xi}[k]\right)=\left\{\begin{array}{l}
\frac{F_{j, 1}\left(\mathbf{a}_{\Xi}^{*}[k]\right)-F_{j, 1}\left(\mathbf{a}_{\Xi}^{* *}[k]\right)}{a_{\xi q}[k]-a_{\xi q}[k-1]} \\
\text { if } \quad a_{\xi q}[k] \not \approx a_{\xi q}[k-1] \\
f_{j}\left(\tilde{\mathbf{a}}_{\Xi}[k]\right) \\
\text { if } \quad a_{\xi q}[k] \approx a_{\xi q}[k-1]
\end{array}\right.
$$

where

$$
\begin{array}{r}
\mathbf{a}_{\Xi}^{*}[k]=\left[\tilde{a}_{\xi 1}[k], \ldots, a_{\xi q}[k], \ldots, \tilde{a}_{\xi J}[k]\right], \\
\mathbf{a}_{\Xi}^{* *}[k]=\left[\tilde{a}_{\xi 1}[k], \ldots, a_{\xi q}[k-1], \ldots, \tilde{a}_{\xi J}[k]\right], \\
\tilde{\mathbf{a}}_{\Xi}[k]=\frac{1}{2}\left(\mathbf{a}_{\Xi}[k]+\mathbf{a}_{\Xi}[k-1]\right),
\end{array}
$$

with

$$
\tilde{a}_{\xi i}[k]=\frac{a_{\xi i}[k]+a_{\xi i}[k-1]}{2},
$$

and $1 \leqslant i \leqslant J$, with $i \neq q$.

It is worth noting that the differences of such an ADAA approach applied to WDFs with one multi-port nonlinearity with respect to the one-port nonlinearity case are confined to how the nonlinear element is handled. In fact the considerations about the temporization of the structure counteracting the additional delay introduced by the ADAA filters in the system feedback loop remain unaltered, and the coefficients of the scattering junctions depending on the sampling period are modified as discussed in the previous section.

As in the nonlinear one-port case it is not always possible to describe multi-port elements using explicit scattering relations like (10), since they may be characterized by a system of implicit nonlinear functions. We will discuss how to manage a multi-port nonlinearity of the sort in Sec. 6, presenting a possible WD ADAA model of the BJT.

\section{HIGHER-ORDER ANTIDERIVATIVE ANTIALIASING IN NONLINEAR WDFS}

The approach described in Sec. 2 can be extended to higher-order ADAA methods to obtain improved aliasing suppression. As before, we consider the one-port nonlinear element case, connected to the same WDF structure of Fig. 1. The nonlinear explicit mapping $b=f(a)$ is now substituted with its generic $p$ th-order ADAA approximation. In [29] a second-order ADAA method is provided, while an alternative general formulation for ADAA of arbitrary-order $p$ is presented in [30]. As an example, second-order ADAA (i.e., $p=2$ ) proposed in [30] and applied to the reference function $f(a)$ yields

$$
\begin{aligned}
\tilde{f}(a[k])= & \frac{2}{a[k]-a[k-2]}\left(\frac{F_{2}(a[k])-F_{2}(a[k-1])}{a[k]-a[k-1]}\right. \\
& \left.-\frac{F_{2}(a[k-1])-F_{2}(a[k-2])}{a[k-1]-a[k-2]}\right),
\end{aligned}
$$

where $F_{2}$ is the second-order antiderivative of $f$. For the treatment of numerical ill-conditioning of (12), possibly occurring when $a[k] \approx a[k-1], a[k] \approx a[k-2]$, or $a[k-$ $1] \approx a[k-2]$, the reader is referred to [30].

In $p$ th-order ADAA, the approximation $\tilde{f}$ of $f$ introduces a delay of $p / 2$ samples [31], causing the waves incident to the junction in vector $\mathbf{b}[k]$ to be misaligned in time. Therefore we need to apply synchronization delays, similarly to what was done in (7). In particular, with higher-orders, the synchronization delays are fractional only when $p$ is odd. For instance if $p=2$ all the waves entering the junction, with the exception of the wave reflected from the nonlinear element, have to be delayed by one sample. To synchronize the signals let us introduce a (potentially) fractional delay of $p / 2$ samples, with Z-domain transfer function $H_{p}(z)$. Although better choices in terms of spectral flatness of the frequency response could certainly be made, in this work we use a simple and computationally lightweight filter with transfer function

$$
H_{p}(z)= \begin{cases}\frac{1}{2}\left(z^{-\left\lfloor\frac{p}{2}\right\rfloor}+z^{-\left(\left\lfloor\frac{p}{2}\right\rfloor+1\right)}\right) & \text { if } p \text { is odd, } \\ z^{-p / 2} & \text { if } p \text { is even. }\end{cases}
$$

If we define $B_{n}(z)$ as the Z-transform of the discrete-time wave signal incident to port $n$ of the WD junction, i.e., $b_{n}[k]$, a synchronized signal $\tilde{B}_{n}(z)$ with $n \neq \xi$ is obtained by applying the filter $H_{p}(z)$,

$$
\begin{aligned}
& \tilde{B}_{n}(z)= \\
& H_{p}(z) B_{n}(z) \text { with } n=1, \ldots, N \text { and } n \neq \xi,
\end{aligned}
$$

where $\tilde{B}_{n}(z)$ is the Z-transform of $\tilde{b}_{n}[k]$. A synchronized version of $\mathbf{b}[k]$ can now be defined as

$$
\tilde{\mathbf{b}}[k]=\left[\tilde{b}_{1}[k], \ldots, \tilde{b}_{M}[k], \tilde{b}_{\xi}[k], \tilde{b}_{M+2}[k], \ldots, \tilde{b}_{N}[k]\right]^{T} .
$$

However, as in the previous section, we still need to compensate for the additional delay of $p / 2$ samples introduced in the feedback path of the WDF by the $p$ th-order ADAA method. Delay compensation is achieved by modifying the coefficients of the system according to the expanded sampling period $\tilde{T}_{\mathrm{s}}=(1+p / 2) T_{\mathrm{s}}$. In fact a delay of $(1+p / 2)$ samples at the reduced sampling frequency $\tilde{f}_{\mathrm{s}}=f_{\mathrm{s}} /(1+p / 2)$ corresponds to a one-sample delay at the reference sampling frequency $f_{\mathrm{s}}$. This translates to the use of a modified scattering junction $\tilde{\mathbf{S}}=\mathbf{S}\left(\tilde{T}_{\mathrm{s}}\right)$ in both the forward and backward scan stage.

In the case of a multi-node CT structure the procedure is analogous, having to adjust the coefficients of each junction according to the expanded sampling period $\tilde{T}_{\mathrm{s}}$. We achieve signal synchronization during the backward scan by applying the $H_{p}(z)$ filter to waves incident to each junction, with the exception of waves incident to ports facing the nonlinear element or other junctions.

The approach discussed in Sec. 2.2 can easily be generalized to integrate $p$ th-order ADAA into WDFs containing one $J$-port nonlinear element by following the considerations provided in this section.

\section{GENERAL WAVE DIGITAL ADAA MODELS OF ONE-PORT NONLINEARITIES}

This section presents a general framework to derive WD ADAA models of one-port nonlinear elements. The general approach presented here will then be employed in Sec. 5 for deriving specific WD ADAA models of diode-based one-port nonlinearities. 
The most general description of an instantaneous nonlinear one-port in the Kirchhoff domain is based on the following equation:

$$
g_{\mathrm{K}}(i, v)=0 .
$$

Often (15) can be made explicit, either obtaining a nonlinear voltage-controlled nonlinearity in the form $i=f_{i}(v)$ or a current-controlled nonlinearity in the form $v=f_{v}(i)$.

When it comes to deriving the WD model of a nonlinear element, depending on the specific nonlinearity and the form of its constitutive equation, it might be convenient to apply the transformation from the Kirchhoff domain to the WD domain using different variable substitution strategies, i.e., directly applying the mapping in Eq. (2) or using intermediate hybrid substitutions involving both Kirchhoff and WD variables. In the following we will show that such different variable substitution choices also lead to different approaches for deriving WD ADAA models of nonlinear one-ports. Similar considerations on the modeling of grouped multiple nonlinearities in WDFs are discussed in [50].

\subsection{ADAA in the Domain of Wave Variables}

In this subsection we consider ADAA approaches that work entirely in the domain of wave variables: by applying the transformation in Eq. (2) to each Kirchoff variable in (15), a nonlinear relation between $a$ and $b$ in the WD domain is obtained, which may or may not be explicit.

Whenever it is possible to obtain an explicit function in the form $b=f(a)$, ADAA can be applied directly according to Eqs. (4) and (12). Otherwise a possible approach consists of tabulating the solution of the resulting implicit nonlinear function $g_{\mathrm{W}}(a, b)=0$ and performing data interpolation at runtime. In order to evaluate ADAA formulas, like (4) and (12), it is also necessary to compute the antiderivatives numerically, starting from the tabulated points. Approaches employing lookup tables have already been shown to be suitable for VA applications and have been extensively used in the literature within the WDF [45, 47] and state-space $[2,51]$ frameworks.

The proposed procedure can be resumed with the following list of steps, where the first three steps are performed offline:

- Store an ordered set of $L$ values for the incident wave $a$ over a certain range and collect them in a vector $\mathbf{a}_{\mathrm{lut}}=\left[a^{(1)}, \ldots, a^{(L)}\right]$.

- For each value $a^{(l)}$ in $\mathbf{a}_{\text {lut }}$, solve the implicit function $g_{\mathrm{W}}\left(a^{(l)}, b^{(l)}\right)=0$ for $b^{(l)}$, employing an iterative solver (e.g., Newton-Raphson), and store the result $b^{(l)}=f\left(a^{(l)}\right)$ in the vector $\mathbf{f}_{\text {lut }}=$ $\left[f\left(a^{(1)}\right), \ldots, f\left(a^{(L)}\right)\right]$.

- Numerically integrate $\mathbf{f}_{\text {lut }}$ with respect to $\mathbf{a}_{\text {lut }}$, obtaining $\mathbf{F}_{\text {lut, } 1}$. The second-order antiderivative $\mathbf{F}_{\text {lut }, 2}$ (and higher-order antiderivatives) can be computed similarly.

- At each discrete-time step $k$, ADAA formulas, like (4) and (12), can be applied on the interpolating func- tions $f(a)=\operatorname{interp}\left(\mathbf{f}_{\text {lut }}, a\right), F_{1}(a)=\operatorname{interp}\left(\mathbf{F}_{\text {lut }, 1}, a\right)$ and $F_{2}(a)=\operatorname{interp}\left(\mathbf{F}_{\text {lut }, 2}, a\right)$, where the generic operator interp simply indicates that the tabulated points are interpolated given the input variable $a$.

\subsection{ADAA in the Hybrid Wave-Kirchhoff Domain}

In many cases given the wave $a$ incident to a nonlinear element it is convenient to evaluate the reflected wave $b$ by using hybrid Wave-Kirchhoff (WK) domain descriptions of the nonlinearity, as they can be easier to handle than the corresponding expressions solely containing wave variables. A hybrid WK description is obtained by expressing solely the port voltage $v$ or port current $i$ in (15) in terms of wave variables, according to one of the following relations $[38,39]$

$$
i=\frac{a-v}{Z}, \quad v=a-Z i .
$$

The result is a nonlinear function in one of the two forms: $g_{\mathrm{Wv}}(v, a)=0$ or $g_{\mathrm{Wi}}(i, a)=0$. Solving $g_{\mathrm{Wv}}(v, a)=0$ for $v$ or $g_{\mathrm{Wi}}(i, a)=0$ for $i$, the reflected wave $b$ can be computed by using one of the following equations

$$
b=2 v-a, \quad b=a-2 Z i .
$$

Dealing with certain nonlinearities it is possible to turn $g_{\mathrm{Wv}}(v, a)=0$ or $g_{\mathrm{Wi}}(i, a)=0$ in explicit form by expressing the port current or port voltage as a function of $a$, i.e., $i=$ $i(a)$ or $v=v(a)$. In such cases we can apply ADAA to those functions. As an example, employing first-order ADAA, the nonlinear functions $v(a)$ and $i(a)$ are approximated by

$\tilde{v}(a[k])=\left\{\begin{array}{lll}\frac{V_{1}(a[k])-V_{1}(a[k-1])}{a[k]-a[k-1]} & \text { if } & a[k] \not \approx a[k-1] \\ v\left(\frac{a[k]+a[k-1]}{2}\right) & \text { if } & a[k] \approx a[k-1]\end{array}\right.$

and

$\tilde{i}(a[k])=\left\{\begin{array}{lll}\frac{I_{1}(a[k])-I_{1}(a[k-1])}{a[k]-a[k-1]} & \text { if } & a[k] \not \approx a[k-1] \\ i\left(\frac{a[k]+a[k-1]}{2}\right) & \text { if } & a[k] \approx a[k-1]\end{array}\right.$

where $V_{1}(a)$ and $I_{1}(a)$ are the first-order antiderivatives of $v(a)$ and $i(a)$, respectively. ADAA application to Eq. (17) hence yields the following antialiased scattering relations

$$
\tilde{b}=2 \tilde{v}-\tilde{a}, \quad \tilde{b}=\tilde{a}-2 Z \tilde{i} .
$$

It is worth noticing that also the incident wave $\tilde{a}$ in (20) has been synchronized with the antialiased functions $\tilde{v}$ and $\tilde{i}$, due to the intrinsic ADAA delay. Signals synchronization is obtained by applying the delay filter $H(z)$, presented in SECS. 2 and 3 , as $\tilde{A}(z)=H(z) A(z)$, with $A(z)$ being the Z-transform of $a$.

In cases in which explicit forms $i=i(a)$ or $v=v(a)$ are not available, given a set of incident waves chosen in a suitable range and stored in the vector $\mathbf{a}_{\text {lut }}$ of length $L$, the implicit functions $g_{\mathrm{Wv}_{\mathrm{v}}}(v, a)=0$ and $g_{\mathrm{Wi}_{\mathrm{i}}}(i, a)=0$ in the hybrid WK domain could be solved iteratively for $i$ or $v$ and the results tabulated. Let us consider, for the sake of brevity, only the case $g_{\mathrm{Wv}_{\mathrm{v}}}(v, a)=0$. Following a procedure similar to that of SEC. 4.1 for the implicit wave mapping, for each value $a^{(l)}$ in $\mathbf{a}_{\mathrm{lut}}, g_{\mathrm{Wv}}(v, a)=0$ is solved for $v$ using an iterative solver and the results are stored in a vector $\mathbf{v}_{\text {lut }}=\left[v\left(a^{(1)}\right), \ldots, v\left(a^{(L)}\right)\right]$. In order to 
apply ADAA, finite sets of values of antiderivatives may be obtained by using numerical integration. We define for instance the vectors containing the values of first-order and second-order antiderivatives of $v$ as $\mathbf{V}_{\text {lut, } 1}$ and $\mathbf{V}_{\text {lut, } 2}$, respectively. It follows that in the first-order case, the unknown nonlinear function $v(a)$ can be approximated by using the ADAA formula (18) by performing interpolation and defining $v(a)=\operatorname{interp}\left(\mathbf{v}_{\text {lut }}, a\right)$ and $V_{1}(a)=\operatorname{interp}\left(\mathbf{V}_{\text {lut, } 1}, a\right)$ similarly to what was done in SEC. 4.1. An analogous approach can be used to apply ADAA to the implicit function $g_{\mathrm{Wi}}(i$, a) $=0$.

The modeling approach described in this section will be applied in the next section to derive different WD ADAA models of one-port diode-based nonlinearities. Even though an exhaustive treatment of general WD ADAA models of multi-port nonlinearities will not be presented in this paper, Sec. 6 will introduce a possible WD ADAA model of the BJT that extends certain concepts related to scalar nonlinearities presented in this section to the vector case.

\section{WAVE DIGITAL ADAA MODELS OF ONE-PORT DIODE-BASED NONLINEARITIES}

\subsection{Explicit Models}

The behavior of a single exponential diode or a pair of identical exponential diodes in antiparallel can be expressed through an explicit WD scattering relation by employing the Lambert $\mathrm{W}$ function, as discussed in [15, 17, 52]. This section revises the aforementioned scattering relations and introduces first and second-order antiderivatives of both wave mappings that can be employed in first and secondorder ADAA.

\subsubsection{Single Diode}

Let us consider the large-signal Shockley diode model, which relates the current $i$ through the exponential p-n junction to the voltage $v$ across it

$$
i=I_{s}\left(e^{\frac{v}{\eta_{t}}}-1\right)
$$

where $I_{s}$ is the saturation current, $V_{t}$ is the thermal voltage, and $\eta$ is the ideality factor. The nonlinear Eq. (21) can be expressed in the WD domain as an explicit mapping $b=$ $f(a)[15,17]$ :

$$
\begin{aligned}
& f(a)=u\left(a, Z, I s, V_{t}, \eta\right) \\
& =a+2 Z I_{s}-2 \eta V_{t} \omega\left(\frac{a+Z I_{s}}{\eta V_{t}}+\log \left(\frac{Z I_{s}}{\eta V_{t}}\right)\right),
\end{aligned}
$$

where $\omega$ indicates the Omega Wright function, defined in terms of the principal branch of the Lambert function $\mathrm{W}_{0}$ as $\omega(x)=\mathrm{W}_{0}\left(e^{x}\right)$ [52]. The first-order antiderivative of (22) is

$$
F_{1}(a)=\frac{a^{2}}{2}+2 Z I_{s} a-\eta^{2} V_{t}^{2} \omega(\phi(a))(2+\omega(\phi(a))),
$$

while the second-order antiderivative reads as follows

$$
\begin{aligned}
& F_{2}(a)=\frac{a^{3}}{6}+Z I_{s} a^{2} \\
& -\frac{\eta^{3} V_{t}^{3}}{6} \omega(\phi(a))\left(12+9 \omega(\phi(a))+2 \omega(\phi(a))^{2}\right)
\end{aligned}
$$

where

$$
\phi(a)=\frac{a+Z I_{s}}{\eta V_{t}}+\log \left(\frac{Z I_{s}}{\eta V_{t}}\right) .
$$

\subsubsection{Pair of Identical Diodes in Antiparallel}

The hard clipping function commonly encountered in distortion and overdrive circuits [2, 15-17] is often implemented through a pair of identical diodes in antiparallel. In the considered WD implementation they are modeled as a single one-port nonlinear element whose $i-v$ characteristic is

$$
i=I_{s}\left[\left(e^{\frac{v}{\eta V_{t}}}-1\right)-\left(e^{\frac{-v}{\eta V_{t}}}-1\right)\right] .
$$

Assuming that only one of the two diodes is conducting at a given time instant $[15,17]$, the nonlinear wave mapping for two identical antiparallel diodes can be approximated as

$$
f(a)=\operatorname{sign}(a) u\left(|a|, Z, I_{s}, V_{t}, \eta\right),
$$

where $\operatorname{sign}(a)$ is the sign function. The first-order antiderivative of (27) is

$$
F_{1}(a)=\frac{a^{2}}{2}+2 Z I_{s}|a|-\eta^{2} V_{t}^{2} \omega(\phi(|a|))(2+\omega(\phi(|a|))) \text {. }
$$

The second-order antiderivative $F_{2}$ of (27) is

$$
\begin{aligned}
F_{2}(a)= & \frac{a^{3}}{6}+Z I_{s} \operatorname{sign}(a) a^{2}-\frac{V_{t}^{3} \eta^{3} \operatorname{sign}(a)}{6} \\
& \times \omega(\phi(|a|))\left(12+9 \omega(\phi(|a|))+2 \omega(\phi(|a|))^{2}\right) .
\end{aligned}
$$

\subsubsection{Pair of Different Diodes in Antiparallel}

Whenever two antiparallel diodes are not identical, it is no longer possible to approximate their behavior as in (27), hence the Lambert W function cannot be exploited for deriving an explicit scattering relation. The proposed solution uses the hybrid WK approach discussed in SEC. 4.2 in conjunction with a one-dimensional Newton-Raphson solver.

Let us consider the two antiparallel diodes $D_{1}$ and $D_{2}$ in Fig. 2, both described according to the Shockley model (21). $D_{1}$ has a saturation current $I_{s 1}$ and ideality factor $\eta_{1}$, while $D_{2}$ has a saturation current $I_{s 2}$ and ideality factor $\eta_{2}$. By applying the KCL we can express the port current $i$ as

$i=i_{D 2}-i_{D 1}=I_{s 2}\left(e^{\frac{v}{\eta_{2} V_{t}}}-1\right)-I_{s 1}\left(e^{\frac{-v}{\eta_{1} V_{t}}}-1\right)$.

By substituting $i=(a-v) / Z$ into (30), an implicit function in the form $g_{W v}(v, a)=0$ is obtained. According to the method discussed in SEC. 4.2, the implicit mapping is solved iteratively at some points $a^{(l)}$, and the result is stored in a 


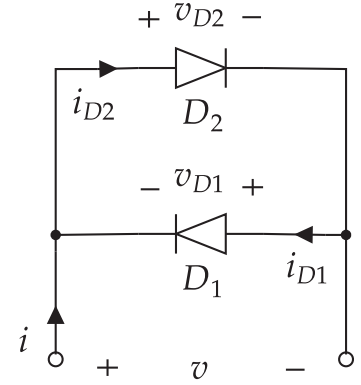

Fig. 2. One-port model of a pair of antiparallel diodes.

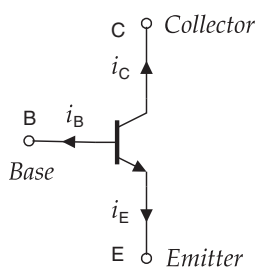

(a)

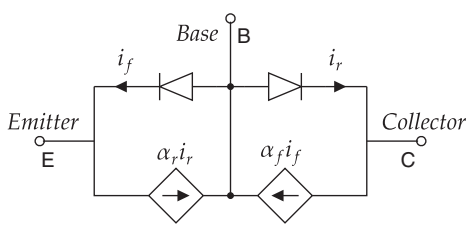

(b)
Fig. 3. Ebers-Moll model.

lookup table. At each point $a^{(l)}$ we can define $h(v)=g_{\mathrm{Wv}}(v$, $\left.a^{(l)}\right)=0$ as

$$
h(v)=v-a^{(l)}+Z I_{s 2}\left(e^{\frac{v}{\eta_{2} V_{t}}}-1\right)-Z I_{s 1}\left(e^{\frac{-v}{\eta_{1} V_{t}}}-1\right),
$$

with derivative

$$
h^{\prime}(v)=1+\frac{Z I_{s 2}}{\eta_{2} V_{t}} e^{\frac{v}{\eta_{2} V_{t}}}+\frac{Z I_{s 1}}{\eta_{1} V_{t}} e^{\frac{-v}{\eta_{1} V_{t}}} .
$$

Equations (31) and (32) are then used to solve $g_{\mathrm{Wv}_{\mathrm{v}}}\left(v, a^{(l)}\right)$ $=0$ for $v$ with the Newton-Raphson method. Finally, the application of ADAA follows the procedure detailed in SEC. 4.2 , and the reflected wave is computed by using the first equation in (20).

\section{MODELING THE BIPOLAR JUNCTION TRANSISTOR (BJT)}

\subsection{BJT Kirchoff Model}

The BJT can be modeled in the Kirchoff domain using the well-known Ebers-Moll model (EMM) [53]. The EMM is depicted in Fig. 3, where the three terminals Base (B), Collector (C), and Emitter (E) are highlighted. The EMM comprises two back-to-back diodes, modeled with the Shockley Eq. (21), and two current-controlled current sources, with $\alpha_{f}$ and $\alpha_{r}$ being the forward and reverse common-base current gains.

The EMM is mathematically expressed as

$$
\left\{\begin{array}{l}
i_{\mathrm{B}}+i_{\mathrm{E}}+i_{\mathrm{C}}=0 \\
i_{\mathrm{E}}=I_{s 1}\left(e^{\frac{v_{\mathrm{BE}}}{n_{1} V_{t}}}-1\right)-\alpha_{r} I_{s 2}\left(e^{\frac{v_{\mathrm{BC}}}{\eta_{2} V_{t}}}-1\right) \\
i_{\mathrm{C}}=I_{s 2}\left(e^{\frac{v_{\mathrm{BC}}}{n_{2} V_{t}}}-1\right)-\alpha_{f} I_{s 1}\left(e^{\frac{v_{\mathrm{BE}}}{\eta_{1} V_{t}}}-1\right) .
\end{array}\right.
$$

\subsection{Generalized WD Model of the BJT}

The WD model of the BJT is obtained following the systematic approach introduced in [21] that allows us to describe any three-terminal element as a WD block characterized by a number of ports $J$ that ranges from 1 to 6 . The choice of the most suitable number of ports $J$ depends on the reference circuit to be implemented as discussed in [21]. The general six-port model of the BJT is shown in Fig. 4, where at each port we connect a Thévenin equivalent circuit. We define two sets of incident waves $\mathbf{a}_{\mathrm{XO}}=\left[a_{\mathrm{BO}}, a_{\mathrm{EO}}, a_{\mathrm{CO}}\right]^{T}$ and $\mathbf{a}_{\mathrm{XY}}=\left[a_{\mathrm{BE}}, a_{\mathrm{EC}}, a_{\mathrm{CB}}\right]^{T}$, with reference port resistance matrices $\mathbf{Z}_{\mathrm{XO}}=\operatorname{diag}\left(\left[Z_{\mathrm{BO}}, Z_{\mathrm{EO}}, Z_{\mathrm{CO}}\right]\right)$ and $\mathbf{Z}_{\mathrm{XY}}=$ $\operatorname{diag}\left(\left[Z_{\mathrm{BE}}, Z_{\mathrm{EC}}, Z_{\mathrm{CB}}\right]\right)$. Moreover we collect all the waves incident to the six-port element in the vector $\mathbf{a}_{\Xi}=$ $\left[\mathbf{a}_{\mathrm{XO}}^{T}, \mathbf{a}_{\mathrm{XY}}^{T}\right]^{T}$.

The implicit nonlinear system of equations in the Kirchhoff variables describing the general six-port model is

$$
\mathbf{g}\left(\mathbf{v}_{K}, \mathbf{a}_{\Xi}\right)=\mathbf{P} \mathbf{f}_{i}\left(\mathbf{v}_{K}\right)+\mathbf{K} \mathbf{f}_{v}\left(\mathbf{v}_{K}\right)+\mathbf{Q \mathbf { a } _ { \Xi }}=\mathbf{0},
$$

where $\mathbf{v}_{K}=\left[v_{\mathrm{BE}}, v_{\mathrm{BC}}\right]^{T}$, with $v_{\mathrm{BC}}=-v_{\mathrm{CB}}$, matrices $\mathbf{P}, \mathbf{K}$, and $\mathbf{Q}$ depend on the chosen number of ports $J$ as discussed in [21], and $\mathbf{0}$ is a $3 \times 1$ vector of zeros. The $3 \times 1$ vector functions $\mathbf{f}_{i}\left(\mathbf{v}_{K}\right)$ and $\mathbf{f}_{v}\left(\mathbf{v}_{K}\right)$ are expressed as a linear combination of two scalar functions of $v_{\mathrm{BE}}$ and $v_{\mathrm{BC}}$, as

$$
\mathbf{f}_{i}\left(\mathbf{v}_{K}\right)=\left[\begin{array}{cc}
\alpha_{f}-1 & \alpha_{r}-1 \\
1 & -\alpha_{r} \\
-\alpha_{f} & 1
\end{array}\right]\left[\begin{array}{l}
f_{\mathrm{BE}}\left(v_{\mathrm{BE}}\right) \\
f_{\mathrm{BC}}\left(v_{\mathrm{BC}}\right)
\end{array}\right]
$$

$$
\mathbf{f}_{v}\left(\mathbf{v}_{K}\right)=\left[\begin{array}{cc}
1 & 0 \\
-1 & 1 \\
0 & -1
\end{array}\right]\left[\begin{array}{l}
v_{\mathrm{BE}} \\
v_{\mathrm{BC}}
\end{array}\right]
$$

with

$$
\begin{aligned}
& f_{\mathrm{BE}}\left(v_{\mathrm{BE}}\right)=I_{s 1}\left(e^{\frac{v_{\mathrm{BE}}}{\eta_{1} V_{t}}}-1\right), \\
& f_{\mathrm{BC}}\left(v_{\mathrm{BC}}\right)=I_{s 2}\left(e^{\frac{v_{\mathrm{BC}} V_{2}}{\eta_{2} V_{t}}}-1\right) .
\end{aligned}
$$

In particular, $\mathbf{f}_{i}\left(\mathbf{v}_{K}\right)$ and $\mathbf{f}_{v}\left(\mathbf{v}_{K}\right)$ are obtained directly from the EMM model. In fact, $\mathbf{f}_{i}\left(\mathbf{v}_{K}\right)=\left[i_{\mathrm{B}}, i_{\mathrm{E}}, i_{\mathrm{C}}\right]^{T}$ equals the currents flowing out of the terminals shown in Fig. 3, while $\mathbf{f}_{v}\left(\mathbf{v}_{K}\right)=\left[v_{\mathrm{BE}}, v_{\mathrm{EC}}, v_{\mathrm{CB}}\right]^{T}$ equals the voltages across the pairs of the BJT terminals.

Since (34) is an implicit system of equations in the variables $v_{\mathrm{BE}}$ and $v_{\mathrm{BC}}$, it is usually solved using the multidimensional Newton-Raphson (MDNR) algorithm [21]. Moreover one of the three equations in (34) is redundant, since it can be expressed as a linear combination of the other two, and is discarded before applying the MDNR method. Removing the redundant equation results in the implicit system of two equations with two unknowns $\mathbf{g}_{\mathrm{nr}}\left(\mathbf{v}_{K}, \hat{\mathbf{a}}\right)=\mathbf{0}$, where $\hat{\mathbf{a}}=\left[\hat{a}_{1}, \hat{a}_{2}\right]^{T}$ contains the remaining two components of the $3 \times 1$ vector $\mathbf{Q \mathbf { a } _ { \Xi }}$, and $\mathbf{0}$ becomes a $2 \times 1$ vector of zeros [21]. Once $v_{\mathrm{BE}}$ and $v_{\mathrm{BC}}$ are computed the waves reflected by the WD element are obtained using the following two expressions

$$
\mathbf{b}_{\mathrm{X} 0}=\mathbf{a}_{\mathrm{X} 0}+2 \mathbf{Z}_{\mathrm{X} 0}\left[\mathbf{f}_{i}\left(\mathbf{v}_{K}\right)+\mathbf{H}^{T} \mathbf{Z}_{\mathrm{XY}}^{-1}\left(\mathbf{a}_{\mathrm{XY}}-\mathbf{f}_{v}\left(\mathbf{v}_{K}\right)\right)\right]
$$




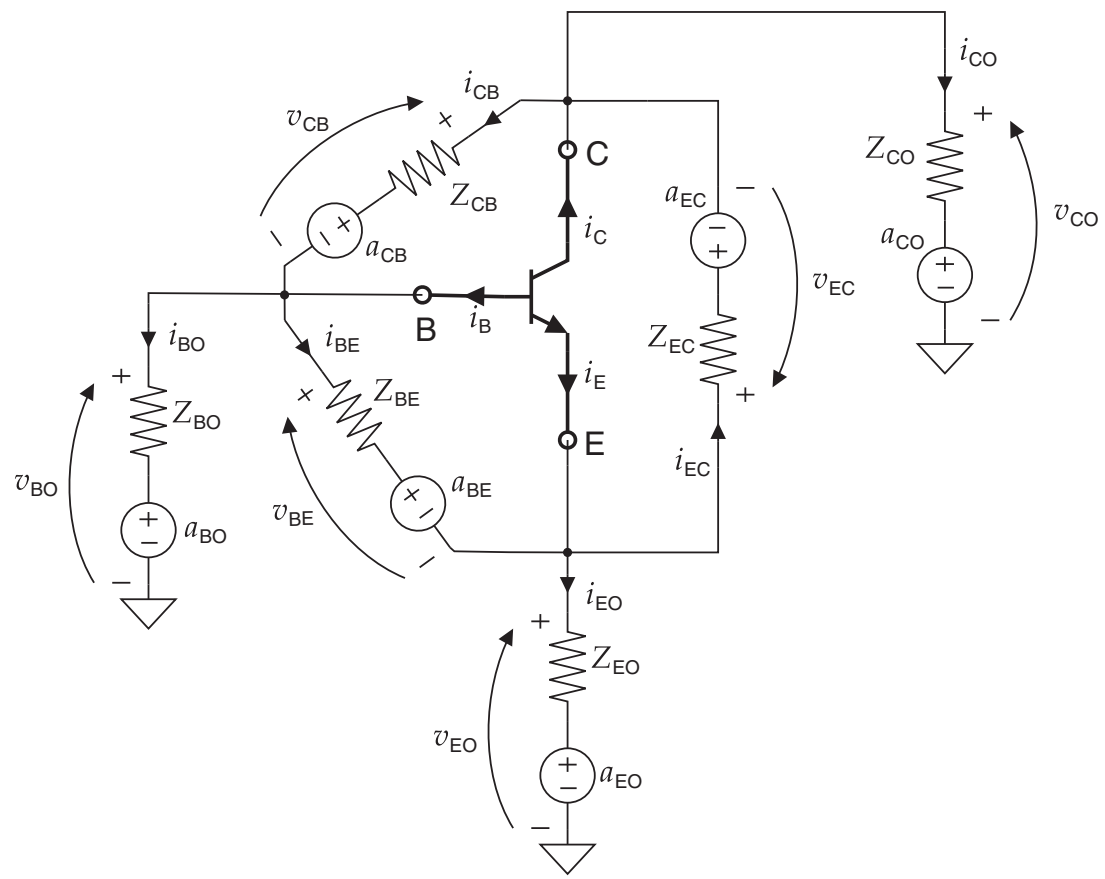

Fig. 4. General six-port WD model of a three-terminal BJT.

$$
\mathbf{b}_{\mathrm{XY}}=2 \mathbf{v}_{\mathrm{XY}}-\mathbf{f}_{v}\left(\mathbf{v}_{K}\right)
$$

where

$$
\mathbf{H}=\left[\begin{array}{ccc}
1 & -1 & 0 \\
0 & 1 & -1 \\
-1 & 0 & 1
\end{array}\right] .
$$

\subsection{Wave Digital ADAA Model of the BJT}

The approach discussed in Sec. 2.2 that applies ADAA to a multi-port nonlinear element characterized by a multivariate vector function is not straightforward, since an integration variable for each vector component needs to be chosen. Fortunately, in some circuits containing BJTs, the nonlinear behavior is dominated by certain variables, making ADAA application to a single integration variable, while keeping the other variables fixed, effective. However the WD BJT model described by the nonlinear implicit equation $\mathbf{g}_{\mathrm{nr}}\left(\mathbf{v}_{K}, \hat{\mathbf{a}}\right)=\mathbf{0}$ prevents us from directly using (11). A possible solution is to use a hybrid WK approach similar to that discussed in Sec. 4.2 but adapted to the multi-port case. In particular, ADAA is applied to the nonlinear functions $f_{\mathrm{BE}}\left(v_{\mathrm{BE}}\right)$ and $f_{\mathrm{BC}}\left(v_{\mathrm{BC}}\right)$. Thus the resulting antialiased versions of the scattering relations (38) and (39) are

$$
\tilde{\mathbf{b}}_{\mathrm{X} 0}=\tilde{\mathbf{a}}_{\mathrm{X} 0}+2 \mathbf{Z}_{\mathrm{X} 0}\left[\tilde{\mathbf{f}}_{i}\left(\mathbf{v}_{K}\right)+\mathbf{H}^{T} \mathbf{Z}_{\mathrm{XY}}^{-1}\left(\tilde{\mathbf{a}}_{\mathrm{XY}}-\tilde{\mathbf{f}}_{v}\left(\mathbf{v}_{K}\right)\right)\right]
$$

$$
\tilde{\mathbf{b}}_{\mathrm{XY}}=2 \tilde{\mathbf{v}}_{\mathrm{XY}}-\tilde{\mathbf{f}}_{v}\left(\mathbf{v}_{K}\right)
$$

where

$$
\tilde{\mathbf{f}}_{i}\left(\mathbf{v}_{K}\right)=\left[\begin{array}{cc}
\alpha_{f}-1 & \alpha_{r}-1 \\
1 & -\alpha_{r} \\
-\alpha_{f} & 1
\end{array}\right]\left[\begin{array}{l}
\tilde{f}_{\mathrm{BE}}\left(v_{\mathrm{BE}}\right) \\
\tilde{f}_{\mathrm{BC}}\left(v_{\mathrm{BC}}\right)
\end{array}\right]
$$

and $\tilde{f}_{\mathrm{BE}}, \tilde{f}_{\mathrm{BC}}$ are the antialiased versions of $f_{\mathrm{BE}}$ and $f_{\mathrm{BC}}$, respectively, while $\tilde{a}_{\mathrm{X} 0}, \tilde{a}_{\mathrm{XY}}, \tilde{\mathbf{f}}_{v}\left(\mathbf{v}_{K}\right)$ are synchronized versions of the respective vector variables, obtained through the linear delay filter $H(z)$ described in Secs. 2 and 3.

To obtain the ADAA approximations $\tilde{f}_{\mathrm{BE}}\left(v_{\mathrm{BE}}\right)$ and $\tilde{f}_{\mathrm{BE}}\left(v_{\mathrm{BC}}\right)$, we derive two lookup tables $\mathbf{f}_{\mathrm{BE} \text {, lut }}$ and $\mathbf{f}_{\mathrm{BC} \text {,lut }}$ mapping â to $f_{\mathrm{BE}}$ and $f_{\mathrm{BC}}$, respectively. The proposed procedure to apply first-order ADAA to the BJT model is reported below. The first three steps of the procedure are performed offline.

- Sample the two components of $\hat{\mathbf{a}}=\left[\hat{a}_{1}, \hat{a}_{2}\right]^{T}$ over suitable ranges, creating a 2D grid of $L \times Q$ points. The resulting grid is characterized by points with coordinates $\left(\hat{a}_{1}^{(l)}, \hat{a}_{2}^{(q)}\right)$, with $1 \leqslant l \leqslant L$ and $1 \leqslant q \leqslant$ $Q$. The created grid will be the input space of the two lookup tables $\mathbf{f}_{\mathrm{BE} \text {,lut }}$ and $\mathbf{f}_{\mathrm{BC} \text {,lut }}$.

- For each $\hat{\mathbf{a}}^{(l, q)}=\left[\hat{a}_{1}^{(l)}, \hat{a}_{2}^{(q)}\right]^{T}$, solve the implicit equation $\mathbf{g}_{\mathrm{nr}}\left(\mathbf{v}_{K}^{(l, q)}, \hat{\mathbf{a}}^{(l, q)}\right)=\mathbf{0}$ for $\mathbf{v}_{K}^{(l, q)}=$ $\left[v_{\mathrm{BE}}^{(l, q)}, v_{\mathrm{BC}}^{(l, q)}\right]^{T}$ using the MDNR and store the values $f_{\mathrm{BE}}\left(v_{\mathrm{BE}}^{(l, q)}\right)$ and $f_{\mathrm{BC}}\left(v_{\mathrm{BC}}^{(l, q)}\right)$ in the two lookup tables $\mathbf{f}_{\mathrm{BE}, \text { lut }}$ and $\mathbf{f}_{\mathrm{BC}, \text { lut. }}$.

- Numerically integrate $\mathbf{f}_{\mathrm{BE} \text {,lut }}$ and $\mathbf{f}_{\mathrm{BC} \text {,lut }}$ with respect to the variable introducing more aliasing (either $\hat{a}_{1}$ or $\hat{a}_{2}$ ), tabulating the values of the first-order antiderivatives in the lookup tables $\mathbf{F}_{\mathrm{BE}, \text { lut, } 1}$ and $\mathbf{F}_{\mathrm{BC} \text {,lut,1. }}$ 


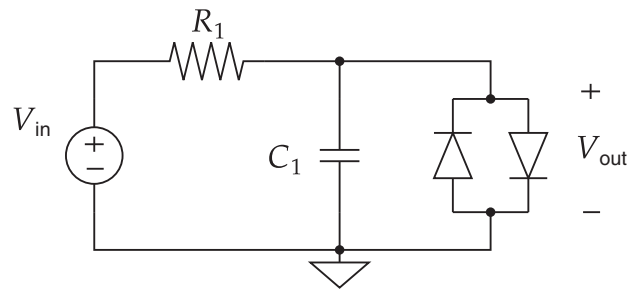

Fig. 5. Circuit schematic of the diode clipper stage.

- At each discrete-time step $k$, ADAA can be applied using an approach mathematically analogous to (11) but employing interpolating functions based on the derived lookup tables.

The generalization of the above procedure to $p$ th-order ADAA is straightforward and only minor adjustments need to be made. In fact $p$ th-order ADAA is obtained by numerically integrating $p$ times the lookup tables $\mathbf{F}_{\mathrm{BE}, \text { lut, } 1}$ and $\mathbf{F}_{\mathrm{BC}, \text { lut,1 }}$ and applying the arbitrary-order formulas given in [30] to (42). Timing issues arising when applying $p$-thorder ADAA to stateful WDFs are handled by using a $p$ th order synchronization filter $H_{p}(z)$, like the one in Eq. (13), and by properly adjusting the coefficients of the modified scattering matrix $\tilde{\mathbf{S}}$, as detailed in Sec. 3 .

\section{EXAMPLES OF APPLICATION}

In this section we describe WDF implementations of three audio circuits and show the effectiveness of the proposed ADAA methods in terms of aliasing reduction. The first WD structure implements an audio clipper circuit and its nonlinearity is a one-port model of a pair of identical antiparallel diodes. The second WD structure implements a modded Tube Screamer clipping stage based on a pair of different antiparallel diodes. The third WD structure implements a common emitter amplifier and it is characterized by a three-port BJT model. In all three digital implementations the reference sampling frequency will be $f_{\mathrm{s}}=44.1$ $\mathrm{kHz}$.

\subsection{Audio Diode Clipper Circuit}

Digital emulations of clipping and limiting musical circuits often generate severe aliasing distortion. This class of waveshaping circuits is commonly encountered in guitar distortion and overdrive effects and many different VA implementations have been discussed in the literature [2, 15-17]. A common example of clipping stage is the circuit shown in Fig. 5. The circuit is composed of an RC filter, with a resistor $R_{1}=1 \mathrm{k} \Omega$ and capacitor $C_{1}=33$ $\mathrm{nF}$, and two antiparallel diodes that "clip" the voltage signals whose amplitude exceeds approximately $\pm 0.7 \mathrm{~V}$. The corresponding WDF realization is shown in Fig. 6. It is characterized by a parallel three-port adaptor $\mathcal{P}_{1}$ that is implemented as in traditional WDFs [35]. The input signal has been modeled through a WD resistive voltage source, using $R_{1}$ as its series resistance. The two antiparallel diodes are grouped together as a one-port nonlinear element placed

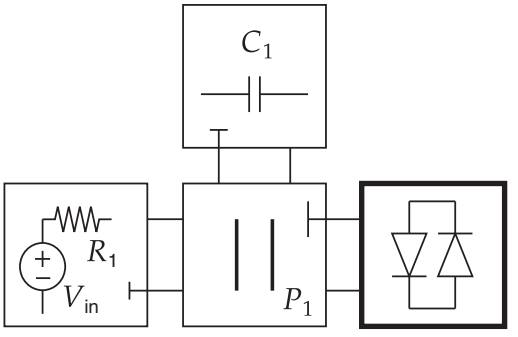

Fig. 6. WDF implementation of the diode clipper.

at the root of the BCT structure and characterized by the explicit relation (27). In particular, first-order ADAA is employed by using (4) in conjunction with (28). Second-order ADAA instead uses (12) along with the first and secondorder antiderivatives (28) and (29). Moreover care must be taken when numerical ill-conditioning occurs, as specified in [30].

The circuit has been tested with a sinusoidal input voltage $V_{\text {in }}(t)=10 \sin \left(2 \pi f_{0} t\right)$ with frequency $f_{0}=1,244.5 \mathrm{~Hz}$. The magnitude spectra of the trivial implementation (i.e., without antialiasing) of the diode clipper circuit as well as those obtained with ADAA filters are shown in Fig. 7. Harmonic components are highlighted with an ' $x$ ' while all the other spikes in the spectrum correspond to aliased components. Fig. 7 shows how ADAA efficiently suppresses aliased components especially at lower frequencies. However it is worth recalling that high-frequency disturbances are often inaudible due to auditory masking effects. Moreover results show that a $\times 2$ oversampling for the ADAA is sufficient to obtain suppression of aliased components comparable to non-antialiased implementations with higher $(x$ 6) oversampling factors, especially employing the secondorder ADAA method.

A possible metric to measure the suppression of the aliased components with respect to desired harmonic distortion components of the clipping stage is the Signal-to-Noise Ratio (SNR), here defined as a power ratio between the desired harmonic components and the aliased components. The SNR analysis has been performed as described in [25, 31] for a set of sinusoidal inputs at different fundamental frequencies, ranging from $1 \mathrm{kHz}$ to $10 \mathrm{kHz}$. For each test signal an ideal alias-free version was obtained by calculating the discrete-time Fourier transform at integer multiples of the fundamental frequency up to the Nyquist frequency and using additive synthesis. The ideal signal was then subtracted from the aliased signal to obtain the residual (i.e., the aliasing distortion). Since in audio applications SNR is only meaningful at audible frequencies all signals were low-pass filtered with cut-off frequency $18 \mathrm{kHz}$ before SNR calculation. In Fig. 8 OS indicates the oversampling factor that multiplies the reference sampling frequency $f_{\mathrm{s}}=44.1$ $\mathrm{kHz}$ and $p$ indicates the ADAA order; trivial refers to the output signal obtained with no ADAA application.

\subsection{Tube Screamer Clipping Stage}

The Tube Screamer is one of the most famous overdrive guitar pedals, characterized by a tube-like distortion and an 

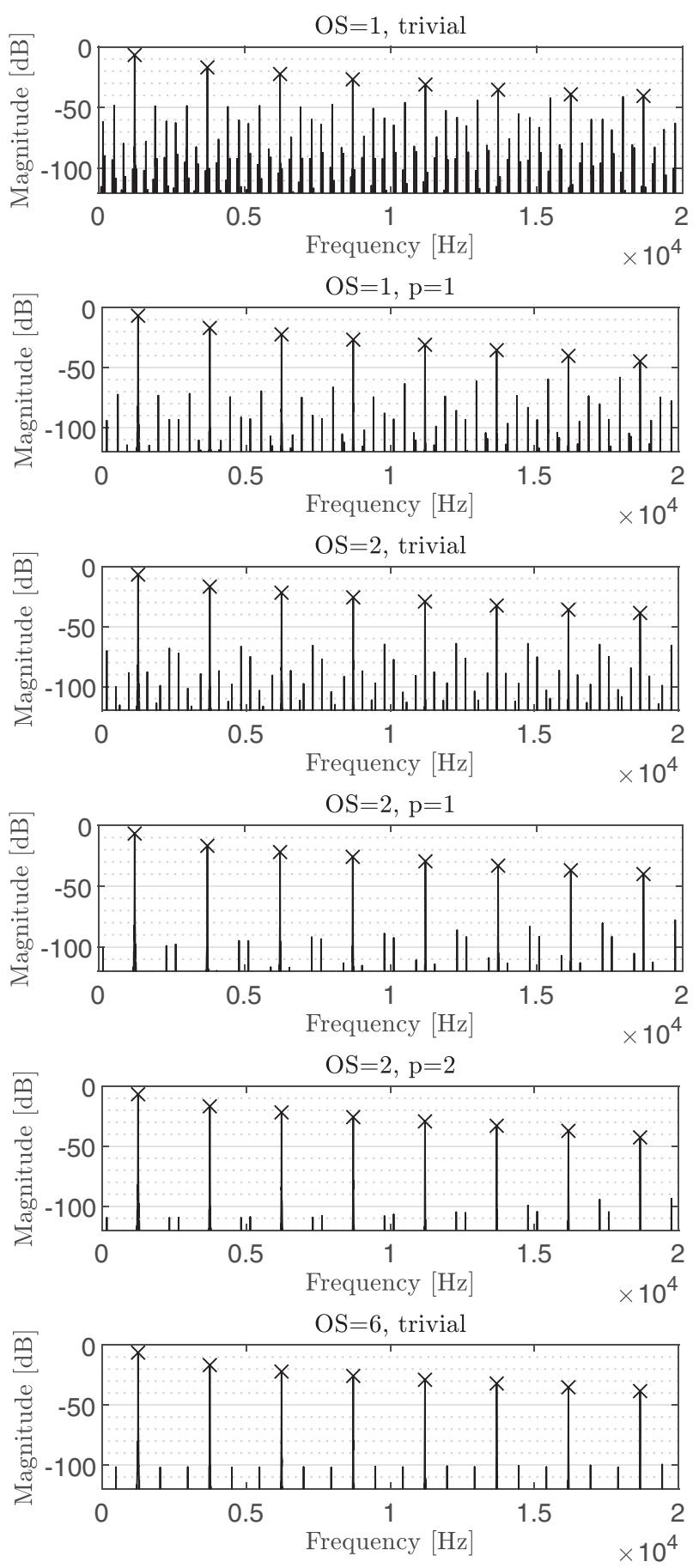

Fig. 7. Diode clipper spectra employing different ADAA orders $p$ and oversampling factors OS, with reference sampling frequency $f_{\mathrm{s}}=44.1 \mathrm{kHz}$ and a sinusoidal input of frequency $1,244.5 \mathrm{~Hz}$ and amplitude $10 \mathrm{~V}$.

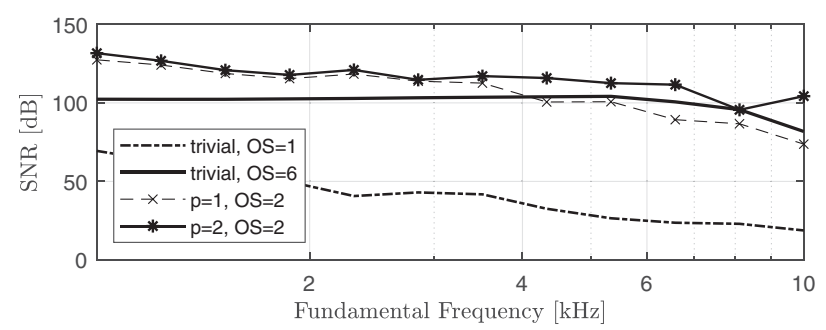

Fig. 8. Diode clipper SNR analysis.

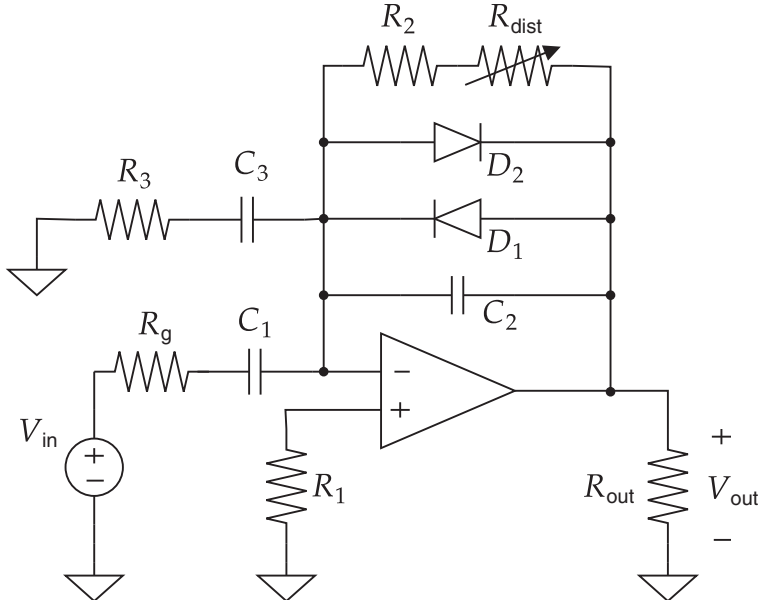

Fig. 9. Circuit schematic of the modded Tube Screamer clipping stage.

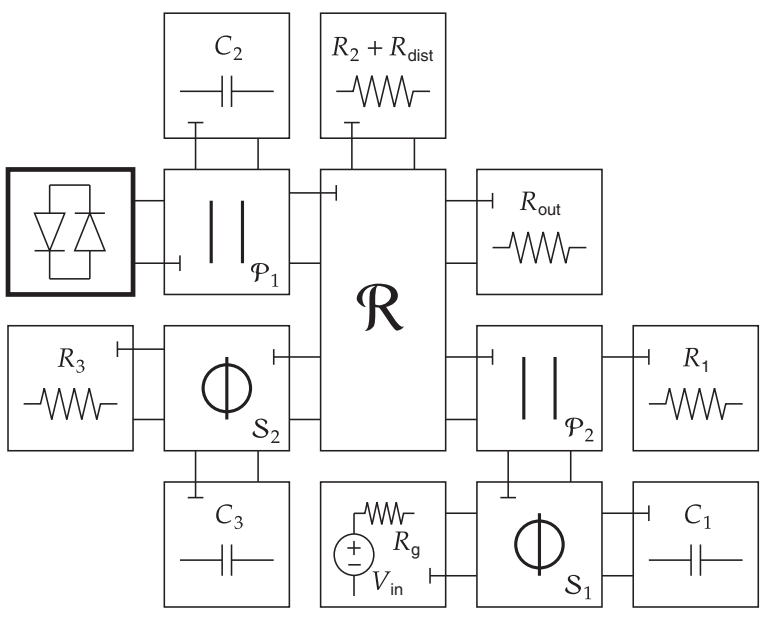

Fig. 10. WDF implementation of the modded Tube Screamer clipping stage.

emphasis on the mid frequencies. The clipping stage of the Tube Screamer is the main responsible for the introduced harmonic distortion. A common mod consists of substituting one of the stock (identical) diodes in the feedback loop with a slightly different one. This process results in asymmetrical clipping and even order distortion.

We propose a WDF implementation of a modified Tube Screamer clipping stage, which employs a 1N914 diode and a 1N4001 diode in an antiparallel configuration. The reference circuit schematic is depicted in Fig. 9 while its WDF implementation is shown in Fig. 10. Circuit parameters are listed in Table 2. In this example the operational amplifier (opamp) has been modeled by using a circuittheoretic two-port element called nullor, following the approach proposed in [38]. The nullor-based opamp model is then absorbed in the non-reciprocal scattering junction $\mathcal{R}$ 
Table 2. Tube Screamer clipping stage parameters.

\begin{tabular}{llll}
\hline \hline Element & Value & Element & Value \\
\hline$R_{1}$ & $10 \mathrm{k} \Omega$ & $C_{1}$ & $1 \mu \mathrm{F}$ \\
$R_{2}$ & $51 \mathrm{k} \Omega$ & $C_{2}$ & $51 \mathrm{pF}$ \\
$R_{\text {dist }}$ & $500 \mathrm{k} \Omega$ & $C_{3}$ & $47 \mathrm{nF}$ \\
$R_{3}$ & $4.7 \mathrm{k} \Omega$ & $I_{s, 1}$ & $2.52 \mathrm{nA}$ \\
$R_{\text {out }}$ & $1 \mathrm{M} \Omega$ & $\eta_{1}$ & 1.752 \\
$R_{g}$ & $0.15 \Omega$ & $I_{s, 2}$ & $2.6 \mu \mathrm{A}$ \\
& & $\eta_{2}$ & 1.6 \\
\hline
\end{tabular}

as explained in [39]. Such a junction is characterized by the following scattering matrix $\mathbf{S}_{\mathcal{R}}$ derived as discussed in [38]

$$
\mathbf{S}_{\mathcal{R}}=\left[\begin{array}{ccccc}
1 & 0 & 0 & 0 & 0 \\
\frac{2\left(Z_{3} Z_{4}+Z_{3} Z_{5}+Z_{4} Z_{5}\right)}{-Z_{5}\left(Z_{3}+Z_{4}\right)} & -1 & \frac{-2 Z_{4}}{Z_{3}+Z_{4}} & \frac{-2 Z_{3}}{Z_{3}+Z_{4}} & \frac{2 Z_{3} Z_{4}}{Z_{5}\left(Z_{3}+Z_{4}\right.} \\
\frac{2 Z_{3} Z_{4}}{Z_{5}\left(Z_{3}+Z_{4}\right.} & 0 & \frac{2 Z_{4}}{Z_{3}+Z_{4}}-1 & \frac{2 Z_{3}}{Z_{3}+Z_{4}} & \frac{-2 Z_{3} Z_{4}}{Z_{5}\left(Z_{3}+Z_{4}\right.} \\
\frac{\left.2 Z_{3} Z_{4}\right)}{Z_{5}\left(Z_{3}+Z_{4}\right)} & 0 & \frac{2 Z_{4}}{Z_{3}+Z_{4}} & \frac{2 Z_{3}}{Z_{3}+Z_{4}}-1 & \frac{\left.-2 Z_{3} Z_{4}\right)}{Z_{5}\left(Z_{3}+Z_{4}\right)} \\
2 & 0 & 0 & 0 & -1
\end{array}\right]
$$

where $Z_{3}=R_{2}+R_{\text {dist }}$ and $Z_{1}, Z_{4}$, and $Z_{5}$ are the free parameters of the adapted ports of the junctions $\mathcal{P}_{2}, \mathcal{P}_{1}$, and $\mathcal{S}_{2}$, respectively. Moreover the adaptation of $\mathcal{R}$ is performed by setting $Z_{4}$ in such a way that the 4 th diagonal entry of $\mathbf{S}_{\mathcal{R}}$ goes to zero (i.e., $Z_{4}=Z_{3}$ ).

Diodes $D_{1}$ and $D_{2}$ are grouped in a single one-port nonlinear element, as done in the implementation of the audio clipper in the previous subsection. Since $D_{1}$ and $D_{2}$ are different, an explicit WD domain scattering relation between the port variables is not available. Hence the WD ADAA model described in SEC. 5.1.3 is used, where $D_{1}$ is the $1 \mathrm{~N} 914$ diode, and $D_{2}$ is the $1 \mathrm{~N} 4001$ diode. The implicit nonlinear function is evaluated offline in order to perform tabulation and considering 1,024 values of $a$ in the range $[-10 ; 10] \mathrm{V}$ and using spline interpolation during the lookup. Fig. 11 shows the aliasing reduction in response to a sinusoidal input of amplitude $0.2 \mathrm{~V}$ and frequency $1,224.5$ $\mathrm{Hz}$ for different combinations of oversampling factors and ADAA orders $p$. Fig. 12 shows the SNR analysis for some sinusoidal inputs of amplitude of $0.2 \mathrm{~V}$ and fundamental frequencies ranging from $1 \mathrm{kHz}$ to $10 \mathrm{kHz}$. The application of ADAA causes an aliasing reduction comparable to the example presented in the previous subsection. Therefore the results suggest that the two different ADAA approaches are comparable in terms of performance.

\subsection{Common Emitter Amplifier}

As a last example we propose a WDF implementation of a common emitter amplifier with a BJT, whose schematic is shown in Fig. 13. Circuit parameters are listed in Table 3 and the resulting WDF structure is shown in Fig. 14. In this example we employ the ADAA technique for multi-port BJTs introduced in SEC. 6.3, employing spline interpolation during the lookup. In particular we model the BJT as a threeport element with ports $\mathrm{BO}, \mathrm{EO}$, and $\mathrm{CO}$. The WD element is described by the implicit system of equations (34), from
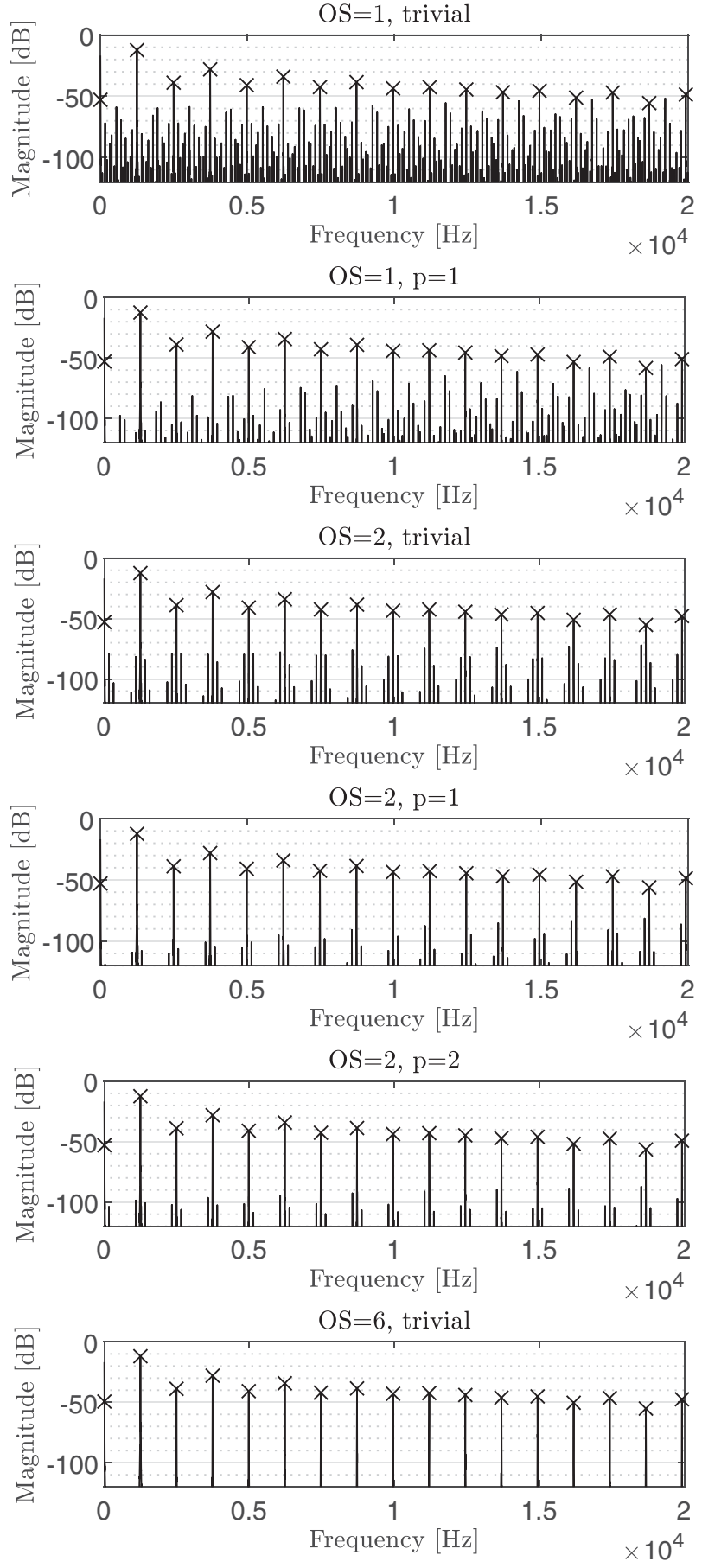

Fig. 11. Tube Screamer clipping stage spectra employing different ADAA orders $p$ and oversampling factors OS, with reference sampling frequency $f s=44.1 \mathrm{kHz}$ and a sinusoidal input of frequency $1,244.5 \mathrm{~Hz}$ and amplitude $0.2 \mathrm{~V}$.

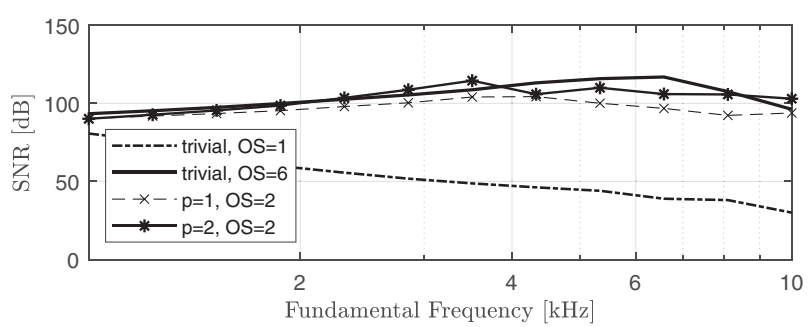

Fig. 12. Tube Screamer clipping stage SNR. 


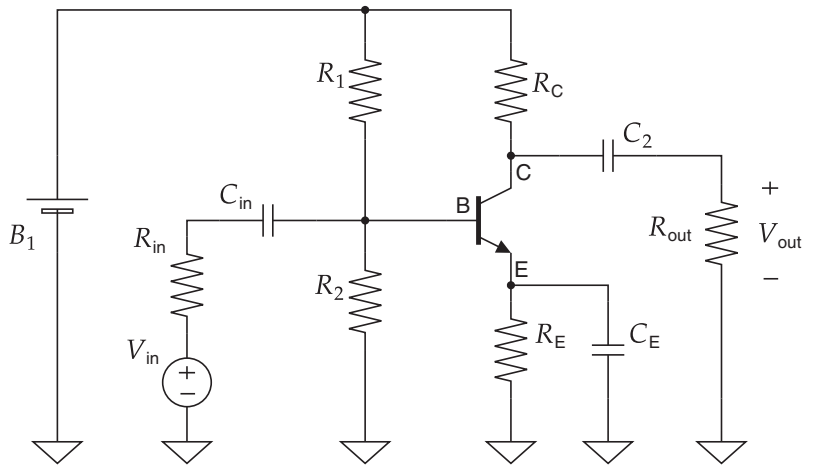

Fig. 13. Circuit schematic of the common emitter amplifier.

Table 3. Common emitter amplifier parameters.

\begin{tabular}{llll}
\hline \hline Element & Value & Element & Value \\
\hline$R_{\text {in }}$ & $1 \mathrm{k} \Omega$ & $C_{\text {in }}$ & $50 \mu \mathrm{F}$ \\
$R_{1}$ & $27.35 \mathrm{k} \Omega$ & $C_{\mathrm{E}}$ & $100 \mu \mathrm{F}$ \\
$R_{2}$ & $2.65 \mathrm{k} \Omega$ & $C_{2}$ & $10 \mu \mathrm{F}$ \\
$R_{\mathrm{E}}$ & $220 \Omega$ & $I_{s 1}$ & $10.05 \mathrm{fA}$ \\
$R_{\mathrm{C}}$ & $1.78 \mathrm{k} \Omega$ & $I_{s 2}$ & $13.33 \mathrm{fA}$ \\
$R_{\text {out }}$ & $1 \mathrm{k} \Omega$ & $\eta_{1}, \eta_{2}$ & 1.5 \\
$B_{1}$ & $18 \mathrm{~V}$ & $\alpha_{r}$ & 0.75 \\
& & $\alpha_{f}$ & 0.995 \\
\hline
\end{tabular}

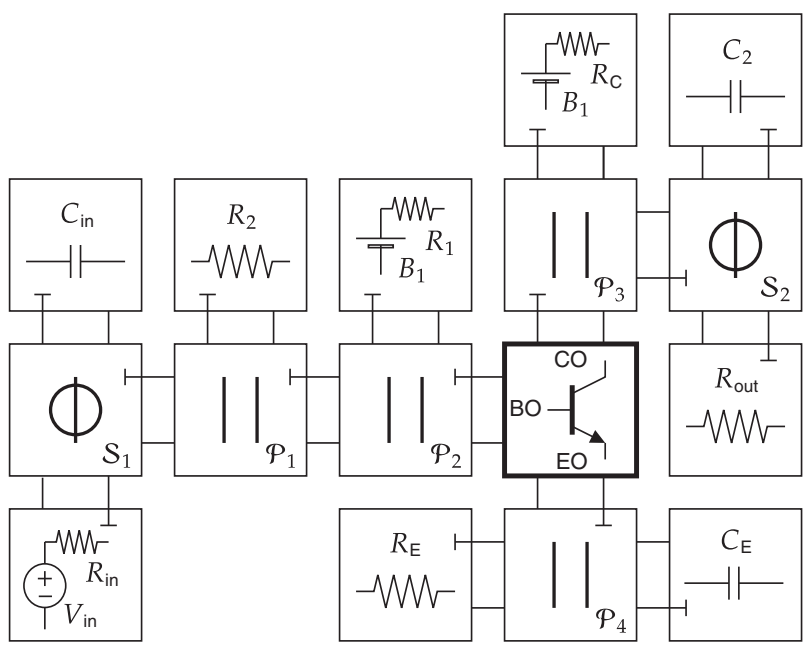

Fig. 14. WDF implementation of common emitter amplifier.

which we remove one redundant equation, obtaining

$$
\begin{aligned}
\mathbf{g}_{\mathrm{nr}}\left(\mathbf{v}_{K}, \hat{\mathbf{a}}\right) & =\left[\begin{array}{ccc}
1 & \frac{-Z_{\mathrm{EO}}}{Z_{\mathrm{BO}}} & 0 \\
0 & 1 & \frac{-Z_{\mathrm{CO}}}{Z_{\mathrm{EO}}}
\end{array}\right] \mathbf{f}_{i}\left(\mathbf{v}_{K}\right)+ \\
& +\left[\begin{array}{ccc}
\frac{-1}{Z_{\mathrm{BO}}} & 0 & 0 \\
0 & \frac{-1}{Z_{\mathrm{EO}}} & 0
\end{array}\right] \mathbf{f}_{v}\left(\mathbf{v}_{K}\right)+\left[\begin{array}{c}
\frac{a_{\mathrm{BO}}}{Z_{B O}}-\frac{a_{\mathrm{EO}}}{Z_{\mathrm{BO}}} \\
\frac{a_{\mathrm{EO}}}{Z_{\mathrm{EO}}}-\frac{a_{\mathrm{CO}}}{Z_{\mathrm{EO}}}
\end{array}\right]=\mathbf{0 .}
\end{aligned}
$$

Moreover only one of the two scattering relations (38) and (39) is necessary and it simplifies to

$$
\mathbf{b}_{\mathrm{X} 0}=\mathbf{a}_{\mathrm{X} 0}+2 \mathbf{Z}_{\mathrm{XO}} \mathbf{f}_{i}\left(\mathbf{v}_{K}\right) .
$$

In the common emitter amplifier circuit the BJT nonlinear behavior is dominated by the variable $\hat{a}_{1}$ that introduces far more aliasing than $\hat{a}_{2}$. This fact allows us to use the methodology described in Sec. 2.2, i.e., integrating both
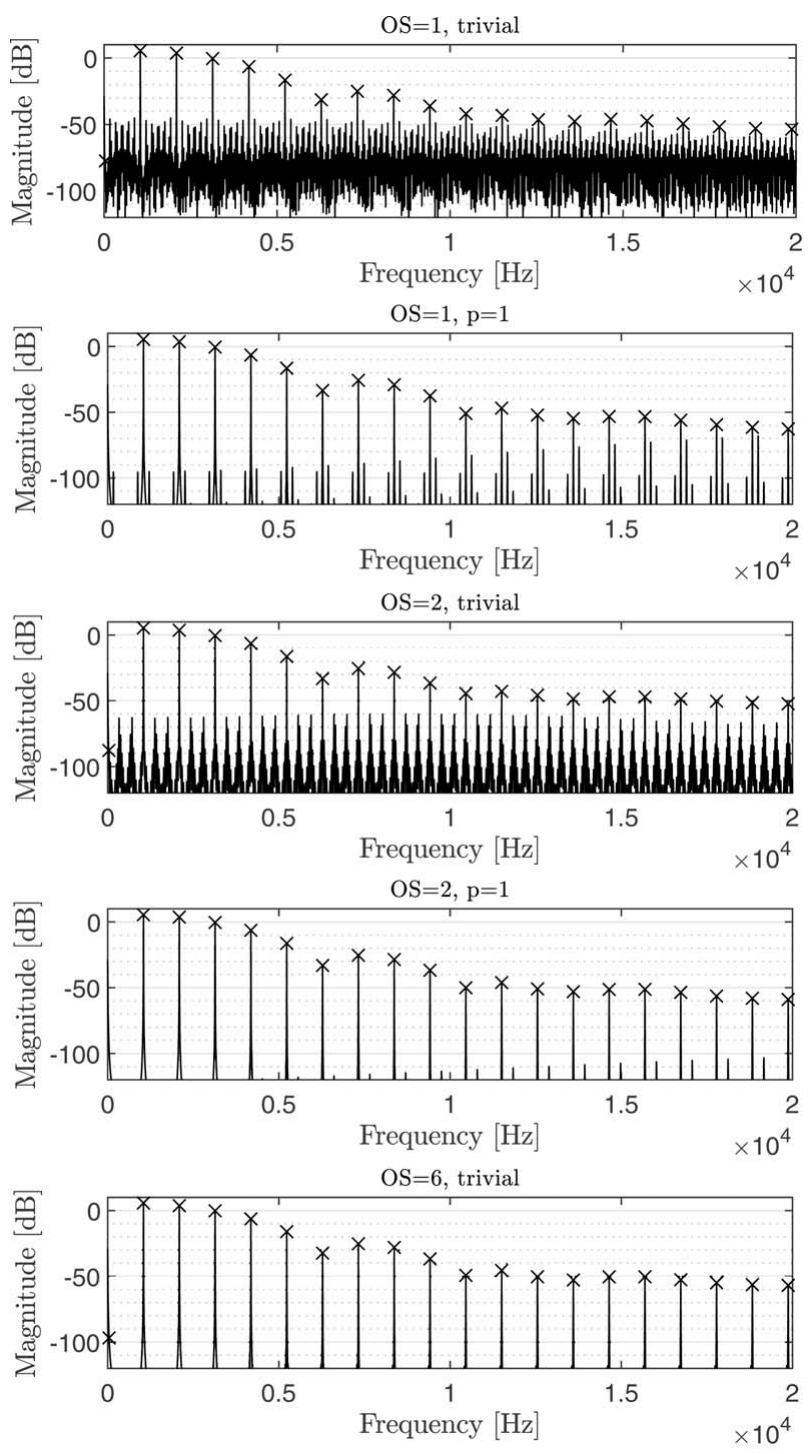

Fig. 15. Common Emitter Amplifier spectra employing different ADAA orders $p$ and oversampling factors OS, with reference sampling frequency $f_{s}=44.1 \mathrm{kHz}$ and a sinusoidal input of frequency $1,046 \mathrm{~Hz}$ and amplitude $0.6 \mathrm{~V}$.

nonlinear functions $f_{\mathrm{BE}}$ and $f_{\mathrm{BC}}$ with respect to $\hat{a}_{1}$, and use the ADAA model described in Sec. 6. Thus ADAA is applied as

$$
\tilde{\mathbf{b}}_{\mathrm{X} 0}=\tilde{\mathbf{a}}_{\mathrm{X} 0}+2 \mathbf{Z}_{\mathrm{XO}} \tilde{\mathbf{f}}_{i}\left(\mathbf{v}_{K}\right) .
$$

The effect of ADAA is shown in Fig. 15, where the spectra of the output signal $V_{\text {out }}$ are obtained by feeding the circuit with a sinusoidal input with frequency $1,046 \mathrm{~Hz}$ (corresponding to a C6 note) and amplitude 0.6 V. Also in this case the results show a significant aliasing reduction; indeed, alias harmonics are almost completely eliminated when first-order ADAA is employed together with an oversampling factor $\times 2$. The results of the SNR analysis are shown in Fig. 16 using sinusoidal inputs with various frequencies ranging from $1 \mathrm{kHz}$ to $10 \mathrm{kHz}$ and with an amplitude of $0.6 \mathrm{~V}$. 


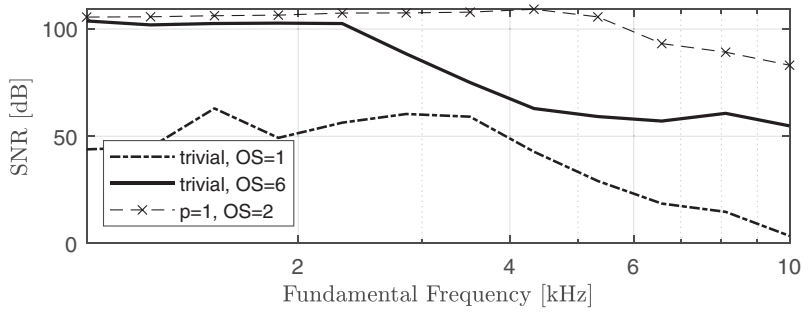

Fig. 16. Common Emitter Amplifier SNR.

\section{CONCLUSIONS AND FUTURE WORK}

In this paper we showed how to integrate arbitrary-order ADAA methods into stateful WDFs with a single one-port or multi-port nonlinear element. The results show good aliasing suppression performance even with low oversampling factors. It is worth noticing that the proposed approach for applying ADAA to digital nonlinear electrical networks fully preserves the modularity properties of traditional WDFs. It is indeed straightforward to change/replace the WD ADAA model of the nonlinear element, with no need for redesigning the rest of the WD structure. On the other hand once an ADAA WD model of a reference nonlinear element (e.g., diode or pair of diodes in antiparallel) has been derived it can be reused for implementing different circuits containing the same nonlinear element.

As far as drawbacks of ADAA filters are concerned it is worth recalling that their response is not spectrally flat, and in particular their inherent low-pass filtering effect could be undesirable. Even if this effect could be easily compensated employing a linear filter, additional spectral shaping could be introduced when the method is applied to stateful systems. The remedy to such spectral shaping is mild oversampling that limits the introduced distortion while maintaining superior antialiasing performance [33].

As a future work we will investigate techniques for the application of ADAA to WD structures with multiple nonlinearities. The problem of managing multiple nonlinearities using ADAA in the WD domain could be approached in different ways. A first approach would be to develop an ADAA model for each nonlinearity and then solve the resulting delay-free-loops. Another possible approach could be to collect all nonlinear elements into a single multi-port nonlinearity and then apply ADAA to such a single multiport nonlinearity, similarly to what we have shown in Sec. 6 discussing the WD ADAA model of the BJT. In both cases a systematic procedure for efficiently solving the resulting system of implicit equations needs to be developed.

\section{REFERENCES}

[1] S. D’Angelo, Virtual Analog Modeling of Nonlinear Musical Circuits, Ph.D. dissertation, Aalto University, Espoo, Finland (2014 Sep.).

[2] D. T. Yeh, J. S. Abel, and J. O. Smith, "Automated Physical Modeling of Nonlinear Audio Circuits for RealTime Audio Effects-Part I: Theoretical Development," IEEE Trans. Audio Speech Lang. Process., vol. 18, no. 4, pp.
728-737 (2010 May). https://doi.org/10.1109/TASL.2009. 2033978.

[3] G. De Sanctis and A. Sarti, "Virtual Analog Modeling in the Wave-Digital Domain," IEEE Trans. Audio Speech Lang. Process., vol. 18, no. 4, pp. 715-727 (2010 May). https://doi.org/10.1109/TASL.2009.2033637.

[4] M. Holters and U. Zölzer, "Physical Modelling of a Wah-Wah Effect Pedal as a Case Study for Application of the Nodal DK Method to Circuits With Variable Parts," in Proceedings of the 14th International Conference on Digital Audio Effects (DAFX-11) pp. 31-35 (Paris, France) (2011 Sep.).

[5] A. Falaize and T. Hélie, "Passive Guaranteed Simulation of Analog Audio Circuits: A Port-Hamiltonian Approach," Appl. Sci., vol. 6, no. 10, pp. 273 (2016 Sep.). https://doi.org/10.3390/app6100273.

[6] R. Müller and T. Hélie, "Power-Balanced Modelling of Circuits as Skew Gradient Systems," in Proceedings of the 21st International Conference on Digital Audio Effects (DAFx-18), pp. 264-271 (Aveiro, Portugal) (2018 Sep.).

[7] E.-P. Damskägg, L. Juvela, and V. Välimäki, "RealTime Modeling of Audio Distortion Circuits With Deep Learning," in Proceedings of the 16th Sound \& Music Computing Conference SMC 2019, pp. 332-339 (Malaga, Spain) (2019 May).

[8] A. Bernardini and A. Sarti, "Towards Inverse Virtual Analog Modeling," in Proceedings of the 22nd International Conference on Digital Audio Effects (DAFx-19) (Birmingham, UK) (2019 Sep.).

[9] A. Huovilainen, "Non-Linear Digital Implementation of the Moog Ladder Filter," in Proceedings of the 7th International Conference on Digital Audio Effects (DAFx'04), pp. 61-64 (Naples, Italy) (2004 Oct.).

[10] F. Fontana and M. Civolani, "Modeling of the EMS VCS3 Voltage-Controlled Filter as a Nonlinear Filter Network," IEEE Trans. Audio Speech Lang. Process., vol. 18, no. 4, pp. 760-772 (2010 May). https://doi.org/10.1109/TASL.2010.2046287.

[11] T. Hélie, "Volterra Series and State Transformation for Real-Time Simulations of Audio Circuits Including Saturations: Application to the Moog Ladder Filter," IEEE Trans. Audio Speech Lang. Process., vol. 18, no. 4, pp. 747-759 (2010 May). https://doi.org/10.1109/TASL.2009. 2035211.

[12] F. Eichas, M. Fink, M. Holters, and U. Zölzer, "Physical Modeling of the MXR Phase 90 Guitar Effect Pedal," in Proceedings of the 17th International Conference on Digital Audio Effects (DAFx-14) (Erlangen, Germany) (2014 Sep.). https://doi.org/10.13140/2.1.3018.9763.

[13] J. Pakarinen and D. T. Yeh, "A Review of Digital Techniques for Modeling Vacuum-Tube Guitar Amplifiers," Comp. Music J., vol. 33, no. 2, pp. 85-100 (2009). https://doi.org/10.1162/comj.2009.33.2.85.

[14] F. Eichas and U. Zölzer, "Gray-Box Modeling of Guitar Amplifiers," J. Audio Eng. Soc., vol. 66, no. 12, pp. 1006-1015 (2018 Dec.). https://doi.org/10.17743/ jaes.2018.0052.

[15] R. C. D. Paiva, S. D’Angelo, J. Pakarinen, and V. Valimaki, "Emulation of Operational Amplifiers and 
Diodes in Audio Distortion Circuits," IEEE Trans. Circuits Syst. II Exp. Briefs, vol. 59, no. 10, pp. 688-692 (2012 Oct.). https://doi.org/10.1109/TCSII.2012.2213358.

[16] K. J. Werner, V. Nangia, A. Bernardini, J. O.III Smith, and A. Sarti, "An Improved and Generalized Diode Clipper Model for Wave Digital Filters," presented at the 139th Convention of the Audio Engineering Society (2015 Oct.), paper 9360.

[17] A. Bernardini, K. J. Werner, A. Sarti, and J. O.III Smith, "Modeling Nonlinear Wave Digital Elements Using the Lambert Function," IEEE Trans. Circuits Syst. I Reg. Papers, vol. 63, no. 8, pp. 1231-1242 (2016 Aug.). https://doi.org/10.1109/TCSI.2016.2573119.

[18] A. Bernardini, K. J. Werner, P. Maffezzoni, and A. Sarti, "Wave Digital Modeling of the Diode-Based Ring Modulator," presented at the 144th Convention of the Audio Engineering Society (2018 May), paper 10015.

[19] A. Bernardini and A. Sarti, "Canonical PiecewiseLinear Representation of Curves in the Wave Digital Domain," in Proceedings of the 25th European Signal Processing Conference (EUSIPCO), pp. 1125-1129 (Kos, Greece) (2017 Aug./Sep.). https://doi.org/10.23919/ EUSIPCO.2017.8081383.

[20] A. Proverbio, A. Bernardini, and A. Sarti, "Toward the Wave Digital Real-Time Emulation of Audio Circuits With Multiple Nonlinearities," in Proceedings of the 2020 28th European Signal Processing Conference (EUSIPCO), pp. 151-155 (Amsterdam, Netherlands) (2021 Jan.). https://doi.org/10.23919/Eusipco47968.2020.9287449.

[21] A. Bernardini, A. E. Vergani, and A. Sarti, "Wave Digital Modeling of Nonlinear 3-Terminal Devices for Virtual Analog Applications," Circuits Syst. Signal Process., vol. 39, pp. 3289-3319 (2020 Jan.). https://doi.org/10.1007/s00034-019-01331-7.

[22] R. C. D. de Paiva, J. Pakarinen, V. Välimäki, and M. Tikander, "Real-Time Audio Transformer Emulation for Virtual Tube Amplifiers," EURASIP J. Adv. Signal Process. (2011 Feb.). https://doi.org/10.1155/2011/347645.

[23] S. D’Angelo, J. Pakarinen, and V. Välimäki, "New Family of Wave-Digital Triode Models," IEEE Trans. Audio Speech Lang. Process., vol. 21, no. 2, pp. 313-321 (2013 Feb.). https://doi.org/10.1109/TASL.2012.2224340.

[24] V. Välimäki and A. Huovilainen, "Antialiasing Oscillators in Subtractive Synthesis," IEEE Signal Process. Mag., vol. 24, no. 2, pp. 116-125 (2007 Mar.). https://doi.org/10.1109/MSP.2007.323276.

[25] V. Välimäki, "Discrete-Time Synthesis of the Sawtooth Waveform With Reduced Aliasing," IEEE Signal Process. Lett., vol. 12, no. 3, pp. 214-217 (2005 Mar.). https://doi.org/10.1109/LSP.2004.842271.

[26] F. Esqueda, V. Välimäki, and S. Bilbao, "Aliasing Reduction in Soft-Clipping Algorithms," in Proceedings of the 23rd European Signal Processing Conference (EUSIPCO), pp. 2014-2018 (Nice, France) (2015 Aug./Sep.). https://doi.org/10.1109/EUSIPCO.2015.7362737.

[27] F. Esqueda, S. Bilbao, and V. Välimäki, "Aliasing Reduction in Clipped Signals," IEEE Trans. Signal Process., vol. 64, no. 20, pp. 5255-5267 (2016 Oct.). https://doi.org/10.1109/TSP.2016.2585091.
[28] J. Kahles, F. Esqueda, and V. Välimäki, “Oversampling for Nonlinear Waveshaping: Choosing the Right Filters," J. Audio Eng. Soc., vol. 67, no. 6, pp. 440-449 (2019 Jun.). https://doi.org/10.17743/jaes.2019.0012.

[29] J. D. Parker, V. Zavalishin, and E. Le Bivic, "Reducing the Aliasing of Nonlinear Waveshaping Using Continuous-Time Convolution," in Proceedings of the 19th International Conference on Digital Audio Effects (DAFx-16), pp. 137-144 (Brno, Czech Republic) (2016 Sep.).

[30] S. Bilbao, F. Esqueda, J. D. Parker, and V. Välimäki, "Antiderivative Antialiasing for Memoryless Nonlinearities," IEEE Signal Process. Lett., vol. 24, no. 7, pp. 1049-1053 (2017 Jul.). https://doi.org/10.1109/LSP.2017. 2675541.

[31] S. Bilbao, F. Esqueda, and V. Valimaki, "Antiderivative Antialiasing, Lagrange Interpolation and Spectral Flatness," in Proceedings of the IEEE Workshop on Applications of Signal Processing to Audio and Acoustics (WASPAA), pp. 141-145 (New Paltz, NY) (2017 Oct.). https://doi.org/10.1109/WASPAA.2017.8170011.

[32] F. Esqueda, H. Pöntynen, J. D. Parker, and S. Bilbao, "Virtual Analog Models of the Lockhart and Serge Wavefolders," Appl. Sci., vol. 7, no. 12, p. 1328 (2017 Dec.). https://doi.org/10.3390/app7121328.

[33] M. Holters, "Antiderivative Antialiasing for Stateful Systems," Appl. Sci., vol. 10, no. 1 (2019 Dec.). https://doi.org/10.3390/app10010020.

[34] D. Albertini, A. Bernardini, and A. Sarti, "Antiderivative Antialiasing in Nonlinear Wave Digital Filters," in Proceedings of the 23nd International Conference on Digital Audio Effects (DAFx), pp. 62-69 (Vienna, Austria) (2020 Sep.).

[35] A. Fettweis, "Wave Digital Filters: Theory and Practice," Proc. IEEE, vol. 74, no. 2, pp. 270-327 (1986 Feb.). https://doi.org/10.1109/PROC.1986.13458.

[36] K. J. Werner, W. R. Dunkel, M. Rest, M. J. Olsen, and J. O. Smith, "Wave Digital Filter Modeling of Circuits With Operational Amplifiers," in Proceedings of the 24th European Signal Processing Conference (EUSIPCO), pp. 1033-1037 (Budapest, Hungary) (2016 Aug./Sep.). https://doi.org/10.1109/EUSIPCO.2016.7760405.

[37] M. Verasani, A. Bernardini, and A. Sarti, "Modeling Sallen-Key Audio Filters in the Wave Digital Domain," in Proceedings of the IEEE International Conference on Acoustics, Speech and Signal Processing, pp. 431-435 (New Orleans, LA) (2017 Mar.). https://doi.org/10.1109/ ICASSP.2017.7952192.

[38] K. J. Werner, A. Bernardini, J. O. Smith, and A. Sarti, "Modeling Circuits With Arbitrary Topologies and Active Linear Multiports Using Wave Digital Filters," IEEE Trans. Circuits Syst. I Reg. Papers, vol. 65, no. 12, pp. 42334246 (2018 Dec.). https://doi.org/10.1109/TCSI.2018. 2837912.

[39] A. Bernardini, K. J. Werner, J. O. Smith, and A. Sarti, "Generalized Wave Digital Filter Realizations of Arbitrary Reciprocal Connection Networks," IEEE Trans. Circuits Syst. I Reg. Papers, vol. 66, no. 2, pp. 694-707 (2019 Feb.). https://doi.org/10.1109/TCSI.2018.2867508. 
[40] A. Bernardini, P. Maffezzoni, A. Sarti, "Linear Multistep Discretization Methods With Variable StepSize in Nonlinear Wave Digital Structures for Virtual Analog Modeling," IEEE Trans. Audio, Speech, Lang. Process., vol. 27, no. 11, pp. 1763-1776 (2019 Nov.). https://doi.org/10.1109/TASLP.2019.2931759.

[41] A. Bernardini, P. Maffezzoni, and A. Sarti, "Vector Wave Digital Filters and Their Application to Circuits With Two-Port Elements," IEEE Trans. Circuits Syst. I Reg. Papers, vol. 68, no. 3, pp. 1269-1282 (2021 Mar.). https://doi.org/10.1109/TCSI.2020.3044002.

[42] K. Meerkötter and R. Scholz, "Digital Simulation of Nonlinear Circuits by Wave Digital Filter Principles," in Proceedings of the IEEE International Symposium on Circuits and Systems, vol. 1, pp. 720-723 (Portland, OR) (1989 May). https://doi.org/10.1109/ISCAS.1989.100452.

[43] A. Bernardini and A. Sarti, "Biparametric Wave Digital Filters," IEEE Trans. Circuits Syst. I Reg. Papers, vol. 64, no. 7, pp. 1826-1838 (2017 Jul.). https://doi.org/ 10.1109/TCSI.2017.2679007.

[44] A. Sarti and G. De Sanctis, "Systematic Methods for the Implementation of Nonlinear Wave-Digital Structures," IEEE Trans. Circuits Syst. I Reg. Papers, vol. 56, no. 2, pp. 460-472 (2009 Feb.). https://doi.org/ 10.1109/TCSI.2008.2001801.

[45] M. Karjalainen and J. Pakarinen, "Wave Digital Simulation of a Vacuum-Tube Amplifier," in Proceedings of the IEEE International Conference on Acoustics Speech and Signal Processing, pp. 153-156 (Toulouse, France) (2006 May). https://doi.org/10.1109/ICASSP.2006. 1661235 .

[46] A. Bernardini, K. J. Werner, A. Sarti, and J. O. Smith, "Modeling a Class of Multi-Port Nonlinearities in Wave Digital Structures," in Proceedings of the 23rd European Signal Processing Conference (EUSIPCO), pp.
664-668 (Nice, France) (2015 Aug./Sep.). https://doi.org/ 10.1109/EUSIPCO.2015.7362466.

[47] K. J. Werner, V. Nangia, J. O. Smith III, and J. S. Abel, "Resolving Wave Digital Filters With Multiple/Multiport Nonlinearities," in Proceedings of the 18th International Conference on Digital Audio Effects (DAFx15) (Trondheim, Norway) (2015 Nov./Dec.).

[48] M. J. Olsen, K. J. Werner, and J. O.III Smith, "Resolving Grouped Nonlinearities in Wave Digital Filters Using Iterative Techniques," in Proceedings of the 19th International Conference on Digital Audio Effects (DAFx-16), pp. 279-286 (Brno, Czech Republic) (2016 Sep.).

[49] A. Carson, Aliasing Reduction in Virtual Analogue Modelling, M.Sc. thesis, University of Edinburgh, Edinburgh, UK (2020 Sep.).

[50] K. J. Werner, M. J. Olsen, M. Rest, and J. D. Parker, "Generalizing Root Variable Choice in Wave Digital Filters With Grouped Nonlinearities," in Proceedings of the 20th International Conference on Digital Audio Effects (DAFx17), pp. 176-183 (Edinburgh, UK) (2017 Sep.).

[51] M. Holters and U. Zölzer, "A k-d Tree Based Solution Cache for the Non-Linear Equation of Circuit Simulations," in Proceedings of the 24th European Signal Processing Conference (EUSIPCO), pp. 1028-1032 (Budapest, Hungary) (2016 Aug./Sep.). https://doi.org/10.1109/EUSIPCO.2016.7760404.

[52] S. D'Angelo, L. Gabrielli, and L. Turchet, "Fast Approximation of the Lambert W Function for Virtual Analog Modelling," in Proceedings of the 22nd International Conference on Digital Audio Effects (DAFx-19) (Birmingham, UK) (2019 Sep.).

[53] J. J. Ebers and J. L. Moll, "Large-Signal Behavior of Junction Transistors," Proc. IRE, vol. 42, no. 12, pp. 17611772 (1954 Dec.). https://doi.org/10.1109/JRPROC.1954. 274797. 
THE AUTHORS

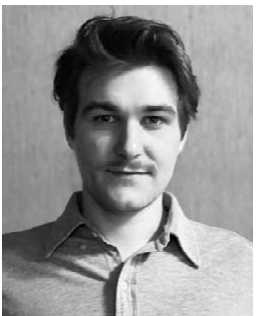

Davide Albertini

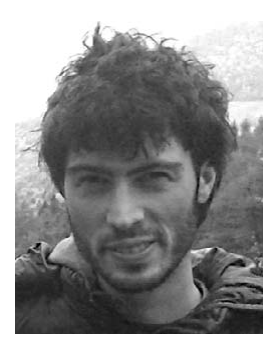

Alberto Bernardini

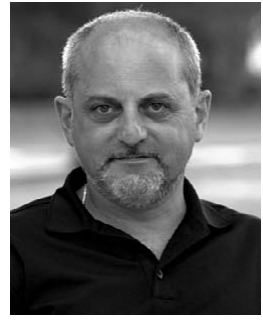

Augusto Sarti
Davide Albertini received his B.S. degree from University of Pavia, Italy in 2016 in Electronics and Computer Engineering and his M.S. degree (cum laude) from Politecnico di Milano, Italy in 2020 in Computer Engineering - Musical Acoustics. He is currently a Ph.D. student at Politecnico di Milano and his main research interests are Virtual Analog modeling of audio circuits and Wireless Acoustic Sensor Networks.

Alberto Bernardini received his B.S. degree from University of Bologna, Italy in 2012 and his M.S. degree (cum laude) from Politecnico di Milano, Italy in 2015, both in Computer Engineering. In 2019 he received his Ph.D. degree (cum laude) in Information Engineering from Politecnico di Milano, Italy, where he is currently a postdoctoral researcher. His main research interests are audio signal processing and modeling of nonlinear systems. He authored over 20 publications in international journals and proceedings of international conferences. He is also the first author of an international patent.

Augusto Sarti received his Ph.D. in Information Engi- neering from the University of Padova, Italy in 1993, with a joint graduate program with the University of California, Berkeley. In 1993 he joined the Politecnico di Milano (PoliMI), Italy, where he is currently a Full Professor. From 2013 to 2017 he held a professorship at the University of California, Davis. At PoliMI he currently coordinates the research activities of the Musical Acoustics Lab and the Sound and Music Computing Lab and the M.Sc. program in "Music and Acoustic Engineering." He has coauthored over 300 scientific publications on international journals and congresses and numerous patents in the multimedia signal processing area. His current research interests are in the area of audio and acoustic signal processing with particular focus on audio and acoustic signal processing, music information retrieval, and musical acoustics. He served two terms with the IEEE Technical Committee on Audio and Acoustics Signal Processing. $\mathrm{He}$ also served as Associate Editor of IEEE/ACM Transactions on Audio Speech and Language Processing and as Senior Area Editor of IEEE Signal Processing Letters. He is currently serving on the EURASIP board of directors. 